\title{
MI-SBTVD: A Proposal for the Brazilian Digital Television System SBTVD
}

\author{
Luciano L. Mendes ${ }^{1}$, José Marcos C. Brito ${ }^{1}$, Fabbryccio A. Cardoso ${ }^{2}$, Dayan A. Guimarães ${ }^{1}$ \\ Gustavo C. Lima ${ }^{3}$, Geraldo G. R. Gomes ${ }^{1}$, Dalton S. Arantes ${ }^{2}$ and Richard D. Souza ${ }^{4}$ \\ ${ }^{1}$ Departamento de Telecomunicações \\ Instituto Nacional de Telecomunicações \\ P.O. Box 05 \\ Phone: +55 (35) 34719200 \\ Zip 37540-000 - Sta. Rita Sapucaí - MG - BRAZIL \\ \{lucianol | brito | dayan |ge|\}@inatel.br \\ ${ }^{2}$ Departamento de Comunicação \\ Universidade Estadual de Campinas \\ P.O.Box 6101, Zip 13083-852 - Campinas - SP - BRAZIL \\ \{fabbryccio|dalton\}@decom.fee.unicamp.br \\ ${ }^{3}$ Grupo de Pesquisa em Comunicação \\ Universidade Federal de Florianópolis \\ Campus Universitário, Florianópolis - SC - BRAZIL \\ guto@eel.ufsc.br Campus Universitário \\ ${ }^{4} \mathrm{CEFET} / \mathrm{PR}$ \\ Curitiba - PR - BRAZIL \\ richard@cpgei.cefetpr.br
}

\begin{abstract}
The objective of this paper is to present a general overview of the Innovative Modulation System Project MI-SBTVD - developed for the Brazilian Digital TV System. The MI-SBTVD Project includes an LDPC high performance error correcting code, an advanced transmit spatial diversity and an efficient multi-carrier modulation scheme. The building blocks of the system, its characteristics and most relevant innovations are presented. The performance of the whole system under different channels is compared with the performance of the present-day Digital Television standards. The complete system was implemented in FPGA using VHDL language and rapid prototyping tools for DSP algorithms.
\end{abstract}

Keywords: Digital Television, LDPC Channel Coding, OFDM Modulation, Spatial Diversity, SBTVD.

\section{INTRODUCTION}

The term "high-definition television" (HDTV) has been used since the late 30 's of the last century to describe a new generation of television system. In that period, the television allowed a monochrome image using 405 lines [1]. The International Consultative Committee for Radio (CCIR), now the International Telecommunications Union - Radiocommunications Sector (ITU-R), defines HDTV in report 801 as [2]: “... a system designed to allow viewing at about three times picture height, such that the system is virtually transparent to the quality of portrayal that would have been perceived in the original scene or performance by a discerning viewer with normal visual acuity". Thus, HDTV is not a digital technology. Maybe, the major turning point to transform HDTV in a digital technology occurred in the USA, in June 1990, with the submission by General Instruments of an all- 
digital proposal to implement an HDTV system. This proposal resulted in a recommendation by the ACATS (Advisory Committee on Advanced Television Systems) Special Panel in March 1993 that only a digital solution should be pursued [1].

The beginning of the standardization process started with the adoption in USA, in 1994, of the ATSC (Advanced Television System Committee) standard. This standard has been developed by a group of companies called Grand Alliance. After that, the DVB-T (Digital Video Broadcasting-Terrestrial) was developed and adopted in Europe and, finally, in 1999, the ISDB-T (Integrated Services Digital Broadcasting - Terrestrial) was adopted in Japan ${ }^{1}$. A brief description of each standard, including their main technical features, is given below.

\subsection{ATSC}

This standard does not support hierarchical transmission, a feature supported by DVB and ISDB. The signal to noise ratio threshold for acceptable quality is around $15 \mathrm{~dB}$ in AWGN (Additive White Gaussian Noise) channel, the best performance of the standards in this kind of channel. On the other hand, the performance of the receiver under dynamic multipath channel is worst when compared with DVB and ISDB. The main characteristics of the ATSC standard, in terms of physical layer, are given in Table 1 [3].

Table 1. Main characteristics of the ATSC standard

\begin{tabular}{|c|c|}
\hline Characteristics & \\
\hline Modulation & 8-VSB (Vestigial Side Band) \\
\hline Inner Code & TCM 2/3 \\
\hline Outer Code & Reed Solomon $(207,187,10)$ \\
\hline Bandwidth & $6 \mathrm{MHz}$ \\
\hline Total Symbol Rate & $10.76 \mathrm{Mbauds}$ \\
\hline Data Bit Rate & $19.28 \mathrm{Mbps}$ \\
\hline
\end{tabular}

\subsection{DVB-T}

This standard has been developed in Europe and it aims at fulfilling the requirements of all the European countries. Thus, the flexibility of the system has been an initial goal of the project.

The main difference between ATSC and DVB-T is in the number of carriers. While ATSC uses a single carrier modulation, DVB-T adopted a multi-carrier solution, using Coded Orthogonal Frequency Division Multiplexing $(\mathrm{COFDM})$ with $2 \mathrm{k}$ or $8 \mathrm{k}$ carriers. The main reason for using this solution is the robustness of this scheme on frequency selective channels.

${ }^{1}$ It is important to observe that there are other standards in the world, like in China, but ATSC, DVB-T and ISDB-T are the most important ones.
Another advantage of the DVB-T, when compared with ATSC, is the hierarchical transmission of up to 2 data streams, which can be used for different applications. This flexibility offers new business models for the TV broadcasters. For example, one option is to use one data stream to broadcast video and audio while the other one may transmit data associated with the scene. This implementation allows an interactivity of the user with the scene, opening new business opportunities. Another possible application is to use one data stream to broadcast SDTV (Standard Definition TV) while the other one is used to transmit the enhanced layer for the HDTV signal. Thus, the users that are capable to receive both streams can watch HDTV, while the users that can receive only the SDTV stream watch the program in standard definition.

The main characteristic of the DVB-T standard, concerning the physical layer, are summarized in Table 2 [4].

Table 2. Main characteristics of the DVB-T standard.

\begin{tabular}{|c|c|}
\hline Characteristics & COFDM \\
\hline Multiplexing & QPSK, 16-QAM or 64-QAM \\
\hline Modulation & Conv. $1 / 2,2 / 3,3 / 4,5 / 6$ or $7 / 8$ \\
\hline Inner Code & Reed Solomon $(204,188,8)$ \\
\hline Outer Code & $6 \mathrm{MHz}, 7 \mathrm{MHz}$ or $8 \mathrm{MHz}$ \\
\hline Bandwidth & $1 / 4,1 / 8,1 / 16$ or $1 / 32$ \\
\hline Guard-time interval & $3.73-23.7 \mathrm{Mbps}$ \\
\hline Data Bit Rate & \\
\hline
\end{tabular}

\subsection{ISDB-T}

This standard has been based on DVB-T, what makes it so similar to the European standard. One advantage of ISDB-T over DVB-T is in an increased flexibility, supported by a new concept for hierarchical transmission based on frequency segmentation. In this case, the total 6 $\mathrm{MHz}$ bandwidth channel is divided in 13 independent segments that can be dynamically grouped to transmit up to 3 different data streams. Table 3 presents the main characteristics of ISDB-T [5].

Table 3. Main characteristics of the ISDB-T standard.

\begin{tabular}{|c|c|}
\hline Characteristics & COFDM \\
\hline Multiplexing & DQPSK, QPSK, \\
Modulation & Conv. 1/2, 2/3, 3/4, 5/6 or $7 / 8$ \\
\hline Inner Code & Reed Solomon $(204,188,8)$ \\
\hline Outer Code & $6 \mathrm{MHz}$ \\
\hline Bandwidth & $1 / 4,1 / 8,1 / 16$ or $1 / 32$ \\
\hline Guard-time interval & $0.28-1.79 \mathrm{Mbps}$ \\
\hline Data Bit Rate / Seg. & \\
\hline
\end{tabular}

As one can see, the currently available Digital Televi- 
sion standards have been conceived in the 90's of the last century. Since then, several important contributions have been proposed for the new generations of digital wireless communications systems. Thus, this is a great opportunity to propose a new standard for Digital Television that includes new technologies, resulting in a system with significant higher capacity and robustness when compared with today's standards.

The goal of this paper is to present a proposal for the Brazilian Digital Television Standard using the state-ofthe-art in communications technologies, offering significantly improved performance when compared with the American, European and Japanese standards.

The remainder of this paper is organized as follows: Section 2 summarizes the main characteristics of the proposed standard; Section 3 presents the characteristics of a mobile channel for digital television. Section 4 shows the options considered and the decisions that have been made by the design team; Section 5 presents the performance of the system in different situations and also compares the MI-SBTVD with other digital television standards; Section 6 shows some implementation issues and the solutions adopted to develop the prototypes. Finally, Section 7 presents the final conclusions.

\section{MI-SBTVD}

In the year 2005, the Brazilian government supported many research consortia in order to develop an advanced Digital Television System employing the most recent technologies for multimedia broadcasting. As part of this development, the MI-SBTVD (Innovative Modulation for the Brazilian Digital Television System) project has proposed a new solution for the physical layer of a new Digital Television standard.

Some guidelines used in the MI-SBTVD project were:

- The SBTVD should have characteristics that facilitate the integration of services, such as e-mail and multimedia services, in order to mitigate the "digital divide" social problem in Brazil.

- The system should have high digital capacity, allowing the transmission of HDTV or multiple programs in SDTV.

- The system should provide mobile reception using in-band transmission. Signals for fixed and mobile receptions should co-exist in the same $6 \mathrm{MHz}$ bandwidth channel. This requirement is important to offer new business models for the broadcasters.

- The capacity and performance of the system should be better than of the former standards.
In order to achieve the goals defined above, the MISBTVD also uses a flexible hierarchical transmission based on frequency segmentation with 13 segments, as in the ISDB-T standard. Some important innovations in wireless communications technologies have been incorporated in the MI-SBTVD project. For example, the inner code has been changed from a convolutional code (used in DVB-T and ISDB-T) to a Low-Density Parity-Check (LDPC) code. Besides, we have used a Space Time Coding (STC) for transmit diversity. LDPC is a very efficient error correction code whose performance is very close to Shannon's limit. Space Time Code is a technique proposed by Alamouti in 1998 that uses up to two transmit antennas and one or multiple receiving antennas to obtain space-time diversity. STC, associated with the OFDM, results in a very robust system for mobile reception on selective channels.

Figures 1 and 2 show the block diagram of the transmitter and of the receiver, respectively. The outer code is the same Reed Solomon $(204,188,8)$ used in DVB-T and ISDB-T. The inner code is an LDPC with codeword length equal to 9792 and code rates $1 / 2,2 / 3,3 / 4,5 / 6$ and 7/8. The modulations are QPSK, 16-QAM and 64-QAM. This set of modulations allows the broadcasters to define the best trade-off between system throughput and robustness. The matrix interleaver between the inner and outer codes improves the performance of the RS decoder.

Table 4 summarizes the main characteristics of the MI-SBTVD system.

Table 4. Main characteristics of the MI-SBTVD system.

\begin{tabular}{|c|c|}
\hline Characteristics & COFDM \\
\hline Multiplexing & DQPSK, QPSK, \\
Modulation & 16-QAM or 64-QAM \\
\hline Inner Code & $3 / 4,5 / 6$ or $7 / 8$ \\
\hline Outer Code & Reed Solomon $(204,188,8)$ \\
\hline Bandwidth & $6 \mathrm{MHz}$ \\
\hline Guard-time interval & $1 / 4,1 / 8,1 / 16$ or $1 / 32$ \\
\hline Diversity & STC-OFDM \\
\hline Data Bit Rate / Seg. & $0.28-1.79 \mathrm{Mbps}$ \\
\hline
\end{tabular}

\section{Channel Characterization}

In this section the main characteristics of the wireless broadcasting DTV (Digital Television) channel are addressed. These characteristics have been determinant in the design and testing phases of the MI-SBTVD system. Further details about them can be found in [6].

When designing a communication system, it is necessary to characterize the channel in order to optimize 


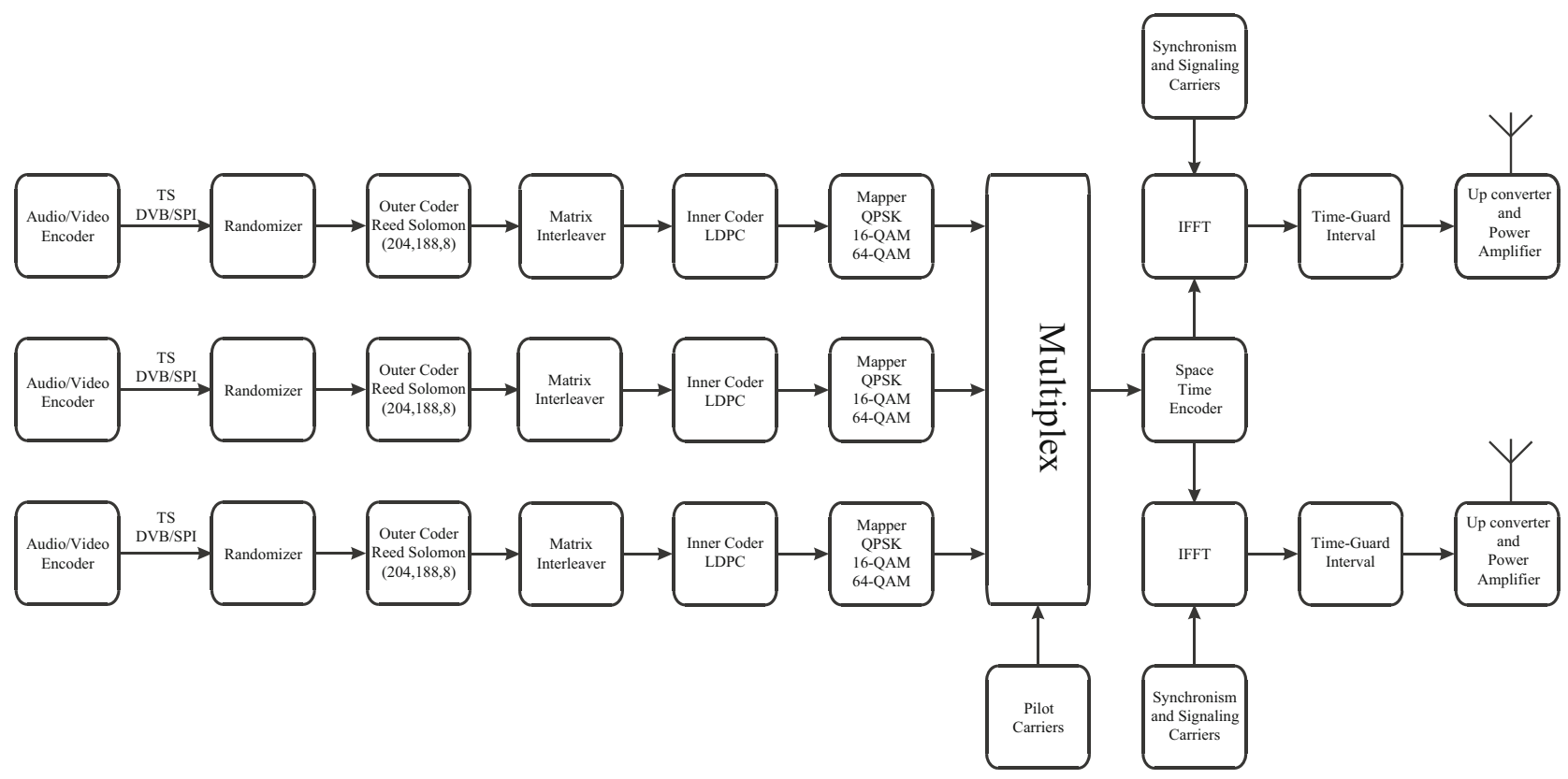

Figure 1. Block diagram of the MI-SBTV transmitter for up to three hierarchical layers.

the overall system. The broadcasting DTV channel can be characterized as a multi-path fading channel in which time dispersion and frequency dispersion may vary with time, depending on the relative motion speed between the transmitter and the receiver. The time dispersion is measured through the time delay profile of the channel and the frequency dispersion is measured through the Doppler profile of the channel.

The time delay profile varies according to the environment. The mobility of the receiver is also important for the system specification, since the speed of the receiver determines the immunity that the system must have to the Doppler spread. Associated with these phenomena, the coherence bandwidth and the coherence time are the main parameters that must be analyzed.

The coherence time of the channel is the time interval in which the channel impulse response can be considered approximately invariant. In other words, it is the time interval where the gain and the phase rotation introduced by the channel are highly correlated. Its value is inversely proportional to the Doppler spread, which is, in turn, directly proportional to the speed of the mobile receiver and the frequency of the signal. The coherence time does not depend on the channel impulse response. Knowledge about it was important to the development of the MI-SBTVD, since it determines the time-selectivity of the channel and, thus, restricts the choice of the modulation to be adopted. It also has influence on the timefrequency interleaver design.
For illustration purposes, Table 5 presents the coherence time for different values of speed and frequency.

The Coherence Bandwidth of a channel is the bandwidth in which the channel frequency response may be considered approximately flat. In other words, it is the bandwidth where the correlation between the magnitude and phase of the channel is high, and it is independent of the speed of the mobile receiver. Knowledge about the coherence bandwidth was important to the development of the MI-SBTVD, since it determines the frequency selectivity of the channel, which determines the spectral characteristics of the signal to be used by the system. It also has influence on the time-frequency interleaver design.

Table 6 shows the delay profiles for DTV static reception and the corresponding coherence bandwidth of the channel [6]. The delay profiles in Table 6 are being used worldwide as reference for designing and testing DTV systems. The channel models Brazil A to Brazil E [7] represent the most common cases in Brazil.

A short description of each channel delay profile is presented as follows:

- UK short delay: describes reception conditions in cases where the terrain is flat.

- UK long delay: describes reception conditions in cases where the terrain has mountains.

- DVB (portable): describes reception conditions of a portable receiver in an urban area. 


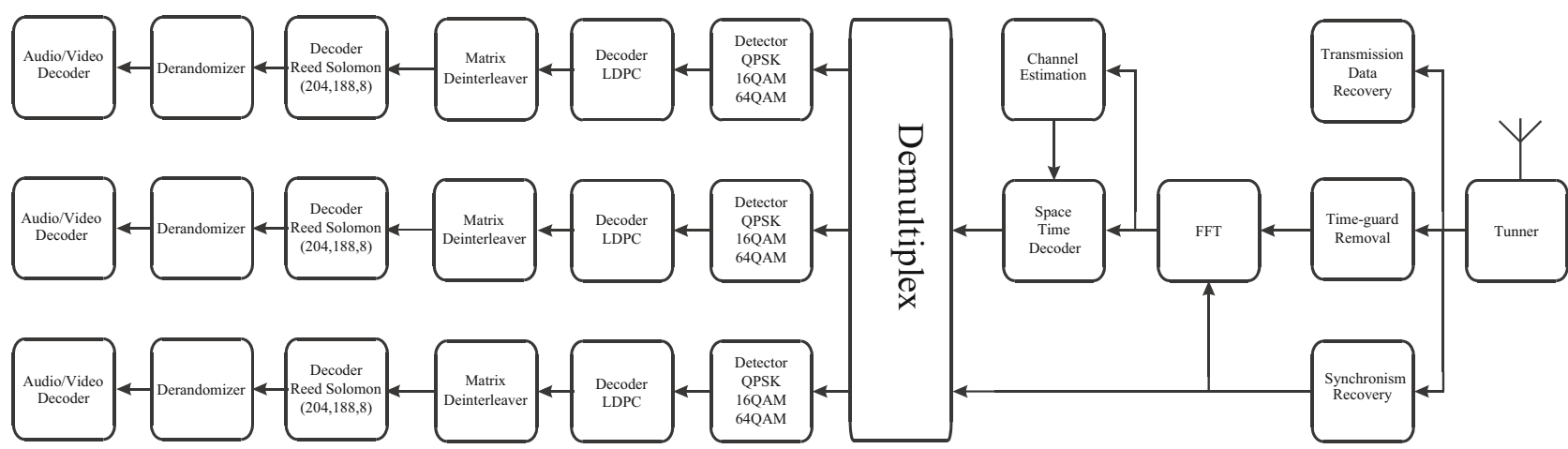

Figure 2. Block diagram of the MI-SBTV receiver for up to three hierarchical layers.

Table 5. Coherence time for different speeds and frequencies.

\begin{tabular}{|c|c|c|c|c|c|}
\hline Freq $/$ Speed & $5 \mathrm{~km} / \mathrm{h}$ & $30 \mathrm{~km} / \mathrm{h}$ & $60 \mathrm{~km} / \mathrm{h}$ & $80 \mathrm{~km} / \mathrm{h}$ & $120 \mathrm{~km} / \mathrm{h}$ \\
\hline $54 \mathrm{MHz}$ & 0.716 & 0.439 & 0.179 & 0.082 & 0.048 \\
$88 \mathrm{MHz}$ & 0.119 & 0.073 & 0.03 & 0.014 & $7 \mathrm{E}-3$ \\
$216 \mathrm{MHz}$ & 0.06 & 0.037 & 0.015 & $6.8 \mathrm{E}-3$ & $4 \mathrm{E}-3$ \\
$470 \mathrm{MHz}$ & 0.045 & 0.027 & 0.011 & $5.14 \mathrm{E}-3$ & $3 \mathrm{E}-3$ \\
$806 \mathrm{MHz}$ & 0.03 & 0.018 & $7.46 \mathrm{E}-3$ & $3.43 \mathrm{E}-3$ & $2 \mathrm{E}-3$ \\
\hline
\end{tabular}

- CRC: this profile represents four different reception conditions that have been used to test ATSC equalizers in Canada.

- Brazil-A: this profile simulates small echoes and short delays. It may represent a channel with lineof-sight in a flat terrain.

- Brazil-B: this profile represents a debilitated reception with external antenna.

- Brazil-C: describes reception conditions in an environment with mountains and no line-of-sight.

- Brazil-D: represents reception conditions with internal antenna.

- Brazil-E: describes reception conditions in a Single Frequency Network environment.

Another channel behavior that is particularly important to design the receiver of a DTV system is the impulsive noise. This kind of impairment is generated in a DTV channel and affects the received signal quality in two main ways: 1) impulsive noise generated by electric power circuitry or through direct induction in the receiver, and 2) impulsive noise captured by the receiver's external antenna. Impulsive noise sources vary from oven ignition systems and fluorescent lamps switching to engine ignition systems. During the design and test of the MISBTVD, we identified impulsive noise types representing worst-case receiver susceptibility for both internal and external receptions.

Another important characteristic of the wireless broadcasting DTV channel, particularly relevant to the MI-SBTVD system design and testing, is the influence of the spatial correlation in the antenna diversity performance. As presented in Section 2, the MI-SBTVD adopted a transmit diversity technique in which the transmitted signal, after an appropriate processing, feeds two antennas. Then, two channels are established from the transmit antennas to the receiver antenna. The efficacy of this diversity scheme is better if the above-mentioned channels are uncorrelated or, at least, have low spatial correlation.

As illustrated in Figure 3, in a wireless broadcasting DTV channel with transmit diversity, the angle $\Delta \varphi$ that embraces the electromagnetic waves capable of hitting the receiver antenna is directly proportional to the radius $a$ in which the scatterers are distributed around the receiver. This angle is inversely proportional to the distance $b$ between the transmitting base-station and the receiver. A typical value for $\Delta \varphi$ is $0.01 \mathrm{rd}$ [6], or $a / b=0.005$. This value is associated with the following scenario: radius of scatterers $a=15 \mathrm{~m}$ and distance between the base-station and the receiver, $b=3 \mathrm{~km}$.

Following [6], we present below the results of two investigations. Figure 4-a shows the spatial correlation $\rho_{r}$ as a function of the ratio between the transmit antenna spacing and the wavelength of the electromagnetic sig- 


\begin{tabular}{|c|c|c|c|c|c|c|c|c|}
\hline Name & $B_{c}[\mathrm{kHz}]$ & Parameter & Path 1 & Path 2 & Path 3 & Path 4 & Path 5 & Path 6 \\
\hline \multirow{3}{*}{ UK Short Delay } & \multirow{3}{*}{18.41} & Delay $(\mu \mathrm{s})$ & 0 & 0.05 & 0.4 & 1.45 & 2.3 & 2.8 \\
\hline & & Atten.(dB) & 2.8 & 0 & 3.8 & 0.1 & 2.6 & 1.3 \\
\hline & & Phase & $0^{\circ}$ & $0^{\circ}$ & $0^{\circ}$ & $0^{\circ}$ & $0^{\circ}$ & $0^{\circ}$ \\
\hline \multirow{3}{*}{ UK Long Delay } & \multirow{3}{*}{4.55} & Delay $(\mu \mathrm{s})$ & 0 & 5 & 14 & 35 & 54 & 75 \\
\hline & & Atten. (dB) & 0 & 9 & 22 & 25 & 27 & 28 \\
\hline & & Phase & $0^{\circ}$ & $0^{\circ}$ & $0^{\circ}$ & $0^{\circ}$ & $0^{\circ}$ & $0^{\circ}$ \\
\hline \multirow{3}{*}{ DVB-T(Portable) } & \multirow{3}{*}{18.19} & Delay $(\mu \mathrm{s})$ & 0.5 & 1.95 & 3.25 & 2.75 & 0.45 & 0.85 \\
\hline & & Atten.(dB) & 0 & 0.1 & 0.6 & 1.3 & 1.4 & 1.9 \\
\hline & & Phase & $336^{\circ}$ & $9^{\circ}$ & $175^{\circ}$ & $127^{\circ}$ & $340^{\circ}$ & $36^{\circ}$ \\
\hline \multirow{3}{*}{$\mathrm{CRC}$} & \multirow{3}{*}{$\operatorname{Var}^{1}$} & Delay $(\mu \mathrm{s})$ & 0 & -1.8 & 0.15 & 1.8 & 5.7 & 35 \\
\hline & & Atten. (dB) & 0 & 11 & 11 & 1 & $\operatorname{Var}^{2}$ & 9 \\
\hline & & Phase & $0^{\circ}$ & $125^{\circ}$ & $80^{\circ}$ & $45^{\circ}$ & $\operatorname{Var}^{1}$ & $90^{\circ}$ \\
\hline \multirow{3}{*}{ Brazil A } & \multirow{3}{*}{13.75} & Delay $(\mu \mathrm{s})$ & 0 & 0.15 & 2.22 & 3.05 & 5.86 & 5.93 \\
\hline & & Atten.(dB) & 0 & 13.8 & 16.2 & 14.9 & 13.6 & 16.4 \\
\hline & & Phase & $0^{\circ}$ & $0^{\circ}$ & $0^{\circ}$ & $0^{\circ}$ & $0^{\circ}$ & $0^{\circ}$ \\
\hline \multirow{3}{*}{ Brazil B } & \multirow{3}{*}{8.98} & Delay $(\mu \mathrm{s})$ & 0 & 0.3 & 3.5 & 4.4 & 9.5 & 12.7 \\
\hline & & Atten.(dB) & 0 & 12 & 4 & 7 & 15 & 22 \\
\hline & & Phase & $0^{\circ}$ & $0^{\circ}$ & $0^{\circ}$ & $0^{\circ}$ & $0^{\circ}$ & $0^{\circ}$ \\
\hline \multirow{3}{*}{ Brazil C } & \multirow{3}{*}{18.43} & Delay $(\mu \mathrm{s})$ & 0 & 0.089 & 0.419 & 1.506 & 2.322 & 2.799 \\
\hline & & Atten.(dB) & 2,8 & 0 & 3.8 & 0.1 & 2.5 & 1.3 \\
\hline & & Phase & $0^{\circ}$ & $0^{\circ}$ & $0^{\circ}$ & $0^{\circ}$ & $0^{\circ}$ & $0^{\circ}$ \\
\hline \multirow{3}{*}{ Brazil D } & \multirow{3}{*}{8.51} & Delay $(\mu \mathrm{s})$ & 0.15 & 0.63 & 2.22 & 3.05 & 5.86 & 5.93 \\
\hline & & Atten.(dB) & 0.1 & 3.8 & 2.6 & 1.3 & 0 & 2.8 \\
\hline & & Phase (Hz) & $0^{\circ}$ & $0^{\circ}$ & $0^{\circ}$ & $0^{\circ}$ & $0^{\circ}$ & $0^{\circ}$ \\
\hline \multirow{3}{*}{ Brazil E } & \multirow{3}{*}{1.91} & Delay $(\mu \mathrm{s})$ & 0 & 1 & 2 & - & - & - \\
\hline & & Atten. (dB) & 0 & 0 & 0 & - & - & - \\
\hline & & Phase & $0^{\circ}$ & $0^{\circ}$ & $0^{\circ}$ & - & - & - \\
\hline
\end{tabular}

${ }^{1}$ Variable according to the Communications Research Centre recommendation.

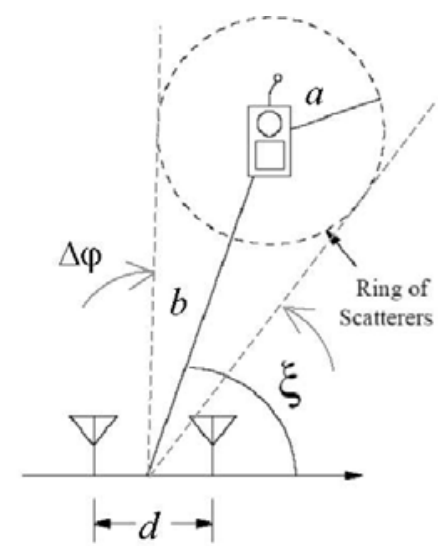

Figure 3. Spatial diversity scenario.

nal, $d / \lambda$, varying $a / b$ and fixing $\xi$. The angle $\xi$ is related to the displacement of the receiver in relation to the line joining the transmit antennas (see Figure 3). Figure 4b shows the spatial correlation $\rho_{r}$ as a function of $d / \lambda$, varying $\xi$ and fixing $a / b$.

It can be seen from Figure 4-a that the spatial correlation $\rho_{r}$ is strongly dependent on the ratio between the radius of scatterers, $a$, and the distance between the basestation and the receiver, $b$. Poor scattering environments (small $a$ ) and high distance $b$ tend to increase the spatial correlation between the signals transmitted from the two antennas to the receiving antenna. Observing Figure 4-b, we can see that the spatial correlation $\rho_{r}$ is also strongly dependent on the angle $\xi$ defined in Figure 3. Receivers located in front of the base-station transmit antennas tend to benefit from lower correlations than receivers located in positions with small $\xi$. The vertical displacement of the transmitting antennas can solve this problem, since $\xi$ will remain practically constant and around $90^{\circ}$. This vertical displacement can also increase the possibilities of having a greater antenna separation $d$. But, unfortunately, in this situation the angle $\Delta \varphi$ decreases, thus reducing the spatial correlation. Coverage problems may also arise from this vertical displacement if the lower antenna is very close to the ground and the upper antenna 


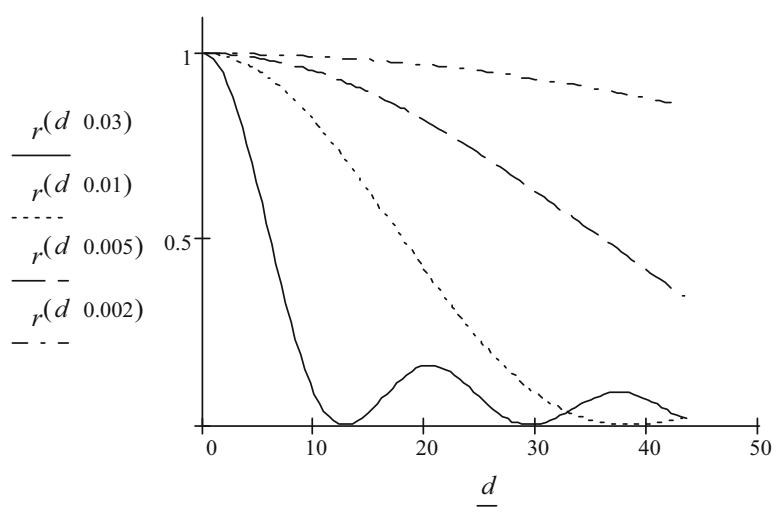

(a)

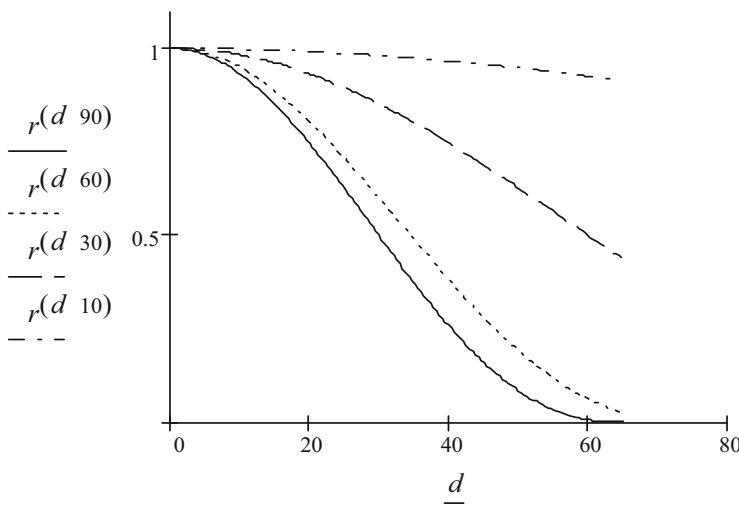

(b)

Figure 4. Spatial correlation $\rho_{r}$ as a function of $d / \lambda$. Varying $a / b$ and $\xi=90^{\circ}$ (a). Varying $\xi$ and $a / b=0.006$ (b).

is in a better situation for coverage. This will demand the use of different transmitting powers in each antenna. The effect of different transmitting powers in the overall system performance should be carefully analyzed.

\subsection{Performance of the Mi-SBTVD Under IMPULSIVE NOISE}

As briefly mentioned in this Section, for testing the MI-SBTVD we identified impulsive noise types representing worst-case receiver susceptibility for both internal and external reception The impulsive noise impairments were generated according to the model depicted in Figure 5 and the parameters given in Table 7, where $\Delta S_{\min }$ is the minimum space between pulses in $\mu \mathrm{s}, \Delta S_{\max }$ is the maximum space between pulses in $\mu \mathrm{s}$ and $S_{\text {effect }}$ is the effective duration in $\mu$ s. Further details can be found in [6].

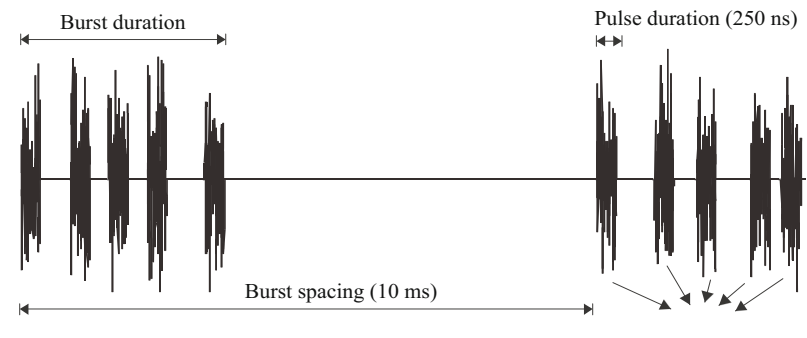

Effective burst duration

Figure 5. Impulsive noise model.

The results concerning the MI-SBTVD impulsive noise immunity, presented in Section V, are interpreted according to Figure 6. In this figure there are five regions,
Table 7. Parameters for Impulsive Noise Testing.

\begin{tabular}{|c|c|c|c|c|}
\hline Test & Pulse / burst & $\Delta S_{\min }{ }^{*}$ & $\Delta S_{\max }{ }^{*}$ & $S_{\text {effec }}$ \\
\hline $\mathrm{A}$ & 1 & N/A & N/A & 0.25 \\
\hline B & 2 & 1.5 & 45 & 0.5 \\
\hline C & 40 & 0.5 & 1 & 10 \\
\hline
\end{tabular}

* Within a burst, the space between pulses are uniformly distributed between the minimum and maximum values specified.

which are described below:

- This asymptotic behavior occurs when the ratio between the signal power and the impulsive noise power $(C / I)$ tends to infinity. It reveals the system performance under AWGN when it reaches the TOV (threshold of visibility).

- When the ratio between the signal power and the AWGN power $(C / N)$ tends to infinity we get the minimum $C / I$ necessary for the system to reach the TOV.

- This intermediate region reveals the system performance under the combined effect of the AWGN and impulsive noise.

- A shift in this curve to the left reveals greater impulsive noise immunity, in terms of $C / I$. The lower bound on this shift corresponds to the value in $\mathrm{dB}$ depicted by the number 5 in the figure.

- A shift in this asymptotic behavior to the bottom reveals improvements in terms of the $C / N$ associated to the TOV. The improvement, in $\mathrm{dB}$, corresponds to the lower bound of the improvement achieved for the impulsive noise, as stated in the previous item. 


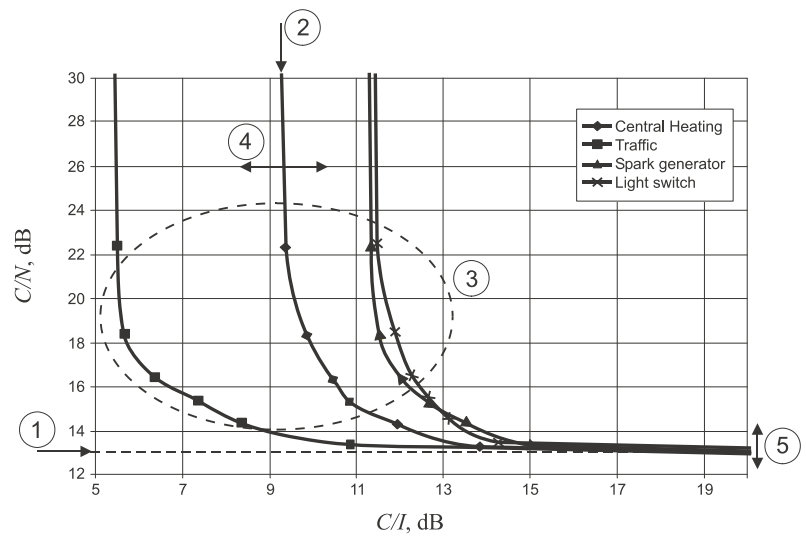

Figure 6. Curves for the MI-SBTVD performance evaluation under AWGN and impulsive noise.

\section{SySTEM DESIGN}

The main objective of the system proposed in the previous section is to obtain higher performance when compared with the Broadcasting DTV standards available today. Besides the performance improvement, the system also needs to guarantee a good trade-off solution between robustness and data rate. This flexibility is important because it allows the TV operators to choose between large coverage with SDTV signal or small coverage with HDTV signal, for example. The next subsections describe each block that composes the system.

\subsection{RANDOMIZER}

The audio and video information, compressed and multiplexed by the audio and video encoders at the MPEG layer, are represented by a bit stream. The Transport Stream (TS) is defined by the MPEG standard as an alternative to transmit the encoded information. In this case, the encoded data is organized in packets of 188 bytes, where the data rate is kept constant by adding null-packets [8]. Figure 7 presents the structure of the TS. The most important information in the TS header for the physical layer is the Synchronism Byte that is $47_{\mathrm{H}}$.

The TS may have a long sequence with certain periodicity, depending on the scene that is being encoded. This periodicity results in spectral concentration, which reduces the performance of the system in selective fading channels, and also may cause synchronism problems at the receiver. In order to avoid the problems associated with long cyclic sequences, the encoded data is multiplied by a pseudo-noise (PN) sequence generated through the polynomial

$$
g(x)=1+X^{14}+X^{15}
$$

The initial seed of the generator is $A 9_{\mathrm{H}}$, and it must be re-initialized at the beginning of each new OFDM frame.

\subsection{Channel Code}

Among the well-know coding channel techniques, a preliminary research suggested the BICM (BitInterleaved Coded-Modulation) as a suitable technique for the SBTVD channel models. The BICM gives best performance on fading channels compared to conventional coded modulations. However, the same does not happens for the AWGN channel, where coded modulations like TCM (Trellis Coded Modulation) gives better results than BICM [9]. BICM was also considered with iterative decoding, or BICM-ID (Bit-Interleaved Coded Modulation with Iterative Decoding), in order to improve the BICM performance on AWGN channels.

In fact, BICM can be interpreted as any coding scheme in which the coded bits are interleaved prior to symbol mapping and modulation. Euclidian distances are not explored as done in conventional coded modulation techniques. The complexity of BICM-ID implementation representes an additional obstacle to the system development in the project schedule. Then, the design team has decided to search for powerful channel coding schemes that could show good performance for both the AWGN and the fading channels. This decision led the team to the capacity-achieving Turbo [10] and LDPC (Low-Density Parity-Check) [11] codes.

The original Turbo codes [10] correspond to the parallel concatenation of recursive and systematic convolutional codes, with a symbol-by-symbol MAP (maximum a posteriori) iterative decoding algorithm. Nowadays, the term Turbo codes has a more generic meaning. Turbo coding is any channel coding technique that uses: 1) an iterative decoding process and 2) a concatenation of interleaved component codes.

Basically, there are two Turbo codes families: the first one is based on a concatenation of convolutional code (CTC, Convolutional Turbo Codes) and the second one is based on a concatenation of block codes (BTC, Block Turbo Codes). Among the BTCs, Single-Parity Check Turbo Product Codes (SPC-TPC) were investigated. The aim of this choice was a reduction in complexity, a fast decoding and, possibly, some performance improvement as compared to high-rate and short convolutional Turbo codes [10].

LDPC codes belong to the class of linear block codes. Their name is due to the parity-check matrix intrinsic characteristic of having a low number of ones, as compared to the number of zeros. The main attribute of LDPC codes is that their performance is also near to the capacity of several communication channels. Besides, the LDPC decoding may be implemented with parallel architecture and relatively low complexity algorithms, as compared to most of the Turbo decoding algorithms [12].

The choice between LDPC and Turbo codes was made based on performance comparisons between Block 


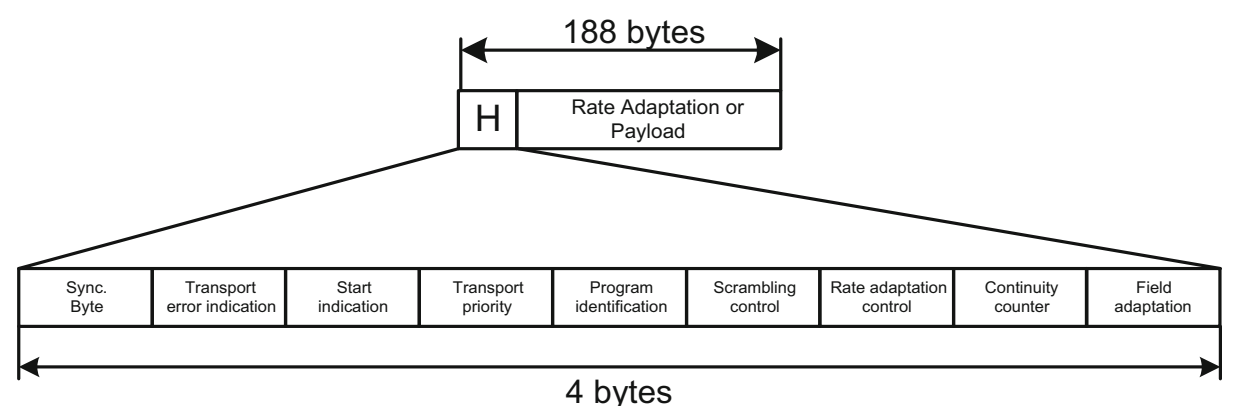

Figure 7. Data structure of the MPEG Transport Stream.

Turbo Codes assembled with Single-Parity Check Product Codes and irregular LDPC codes. These comparisons were carried out by means of computer simulations on AWGN and flat Rayleigh fading channels. The results obtained showed that the LDPC codes produce better performance compared to Turbo codes when the BER (Bit Error Rate) is above the LDPC error floor. The choice in favor of the LDPC code was made based on the reasons: better performance than the SPC-TPC codes; great design flexibility and not too big decoding complexity. Besides, LDPC codes require almost no royalties to be paid, as compared to Turbo codes.

The LDPC error floor can be reduced by the concatenation of the LDPC with some other outer coding scheme. The Reed-Solomon (RS) channel coding was the choice, since it is efficient and can be decoded with low complexity.

With the code already defined, the next step was the choice of the LDPC length suitable to the SBTVD segmentation. The segmentation adopted by the SBTVD OFDM (Orthogonal Frequency-Division Multiplexing) was the same as the one adopted by the ISDB-T system (ARIB STD-B31 V1.5) [5]. This choice was justified by the fact that the project schedule did not allow us to evaluate the impact of a new segmentation on the system implementation and performance. The combination of the ARIB STD-B31 V1.5 segmentation, the OFDM frame structure and the RS $(204,188)$ was determinant on the possible LDPC lengths. Besides, different LDPC coding rates were considered in order to optimize the performance with regards to the segmentation and the channel models. The coding rates $1 / 2,2 / 3,3 / 4,5 / 6$ e $7 / 8$ were defined. The LDPC lengths and the average error correction capability for a $1 / 2$ coding rate are shown in Table 8 .

Initially, the LDPC with code length 39168 was investigated as a possible and efficient solution to eliminate the channel interleaver and, consequently, to simplify the hardware. In fact, computer simulations on the SBTVD channels, including impulsive noise impairments, indeed
Table 8. LDPC lengths and their error correction capability for coding rate $\mathrm{R}=1 / 2$

\begin{tabular}{|c|c|}
\hline Lenght $n$ (bits) & Average error correction $t$ (bits) \\
\hline 39168 & 5162 \\
\hline 19584 & 2520 \\
\hline 13056 & 1645 \\
\hline 9792 & 1211 \\
\hline 6528 & 787 \\
\hline 4896 & 573 \\
\hline 2304 & 241 \\
\hline
\end{tabular}

showed that the channel interleaver was not necessary [12]. However, the external code RS $(204,188)$ cascaded with the $n=39168$ LDPC did not reduce the error floor as expected. This was caused because the LDPC residual errors exhibited frequent burst patterns, exceeding the RS error correction capability.

The trade-off solution between the interleaver elimination and the error-floor reduction was the adoption of the classic solution: an interleaver between the RS (204, 188 ) and the LDPC encoders. However, to avoid a long delay in the decoding process, an LDPC with $n=9792$ was chosen. Since the interleaver length depends on the modulation, different lengths were adopted, according to Table 9.

Table 9. Possible interleaver lengths depending on the modulation.

\begin{tabular}{|c|c|}
\hline Modulation & Interleaver Length \\
\hline QPSK & 4 LDPC words \\
\hline 16-QAM & 8 LDPC words \\
\hline 64-QAM & 12 LDPC words \\
\hline
\end{tabular}

The number of LDPC words has been chosen to allow independency among segments. For example, in mode 1, a segment has 96 data carriers. Using QPSK mapping, this segment can carry 192 data bits. Thus, in a OFDM frame composed of 204 OFDM symbols, this segment is capable of carrying 39168 bits or 4 LDPC words. The 
same reasoning can be applied to 16- and 64-QAM modulations.

The number of data bytes necessary to compose a LDPC word depends on the code rate used. Table 10 shows the number of data bits and data bytes necessary to form an LDPC word.

Table 10. Number of data bits or byte to compose a LDPC w
depending on the code rate.
\begin{tabular}{|c|c|}
\hline Code Rate & Number of Bits and Bytes \\
\hline $1 / 2$ & 4896 bits or 612 bytes \\
\hline $2 / 3$ & 6528 bits or 816 bytes \\
\hline $3 / 4$ & 7344 bits or 918 bytes \\
\hline $5 / 6$ & 8160 bits or 1020 bytes \\
\hline $7 / 8$ & 8568 bits or 1071 bytes \\
\hline
\end{tabular}

\subsection{Transmit Diversity USING Space-Time CODING}

Television broadcasters are very interested in expanding their business model to mobile television, especially for cellular reception. Therefore, the Digital Television system must provide robustness for mobile reception, so the impact of the Doppler spread on the system performance must be minimized. Receivers with spacial diversity [13] could be a solution to this problem. Nevertheless, for a digital television broadcasting system, to keep costs and complexities only in the transmitter, it is more interesting to provide transmit diversity instead of reception diversity, since only the transmitter may have multiple antennas.

In 1998, Alamouti proposed a transmit diversity technique using space-time coding [14]. In this scheme, two transmitting antennas and $L$ receiver antennas can be used to obtain a diversity gain of order $2 L$. However, the communication channel must be characterized by flat, nonselective fading. It is also assumed that the coherence time of the channel is greater than the duration of two consecutive modulation symbols. Moreover, the receiver must perfectly know the channel in order to obtain full diversity gain. However, high transmission rates associated with the mobility of the receivers result in a frequency selective, time-variant fading channel. The conditions above are sufficient to guarantee transmit diversity when the Alamouti scheme is associated with the OFDM technique.

4.3.1. OFDM: Digital data broadcasting channels usually present multiple paths between the transmitting and the receiving antennas [15]. In this scenario, high data rate signals suffer from frequency selective fading.

The basic principle of OFDM transmission technique is to divide the high rate stream into $N$ lower rate streams.
This procedure may transform the original selective fading channel into several flat fading channels. Each lower symbol rate stream is transmitted using a different subcarrier. In order to maximize the spectral efficiency, the sub-carriers are mutually orthogonal. The orthogonality is achieved when the frequency separation between two adjacent sub-carriers is equal to the symbol rate of each sub-stream.

The OFDM symbol can be generated using the Inverse Fast Fourier Transform (IFFT). The sampled version of the OFDM symbol is given by [16]

$$
s_{m}=\sum_{k=0}^{N-1} c_{k} \exp \left(j \frac{2 \pi k}{N} m\right)
$$

where $c_{k}=i_{k}+j q_{k}$ is the vector of complex data symbols and $m=0,1,2, \ldots, N-1$ is the discrete sample time of the OFDM signal.

Using Eq. (2) it is possible to generate OFDM signals by using computational methods, avoiding the synchronism problems with oscillators. Figure 8 shows the block diagram of an OFDM system.

A guard-time interval is used to increase the robustness of the system. The MI-SBTVD system employs a cyclic prefix that is formed by part of the the OFDM symbol which is copied to its beginning, as can be seen in Figure 9.

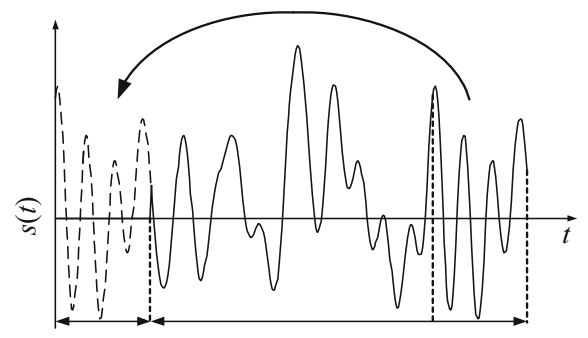

Figure 9. Guard time interval for OFDM symbols.

In the MI-SBTVD system, it is possible to define a cyclic prefix of $1 / 4,1 / 8,1 / 16$ or $1 / 32$ of the OFDM symbol time as a guard-time interval.

4.3.2. Transmit Diversity: Space-Time Block Code (STBC) is a simple solution to obtain diversity gain using two transmitting antennas instead of two receiving antennas [14]. This solution is interesting for digital data broadcasting because the broadcasters are interested in providing mobile services for cellular phones and vehicles. The fact that only the transmitter must have two antennas in order to provide diversity gain makes this technique economically suitable. 


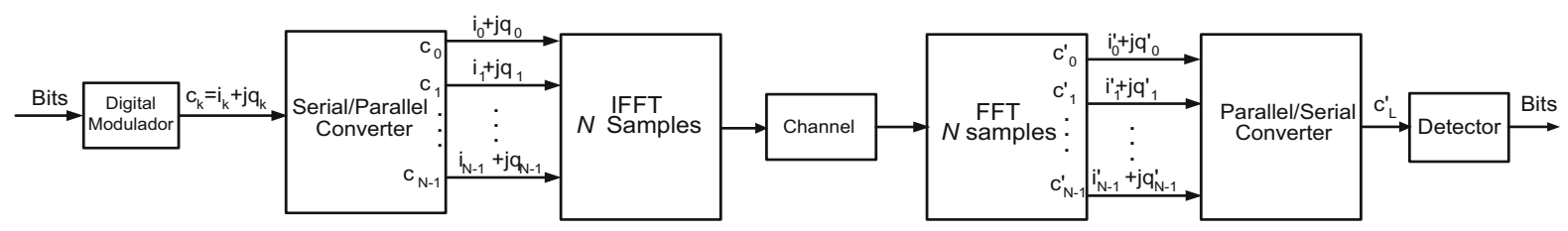

Figure 8. Block diagram of an OFDM system.

STBC uses the transmission matrix given by

\begin{tabular}{c|cc} 
& Ant1 & Ant2 \\
\hline$t_{i}$ & $c_{i}$ & $-c_{i+1}^{*}$ \\
$t_{i+1}$ & $c_{i+1}$ & $c_{i}^{*}$
\end{tabular}

This transmission matrix combines the signals transmitted by both antennas, increasing the performance of the system in flat, time-variant channels. Figure 10 shows the block diagram of this system.

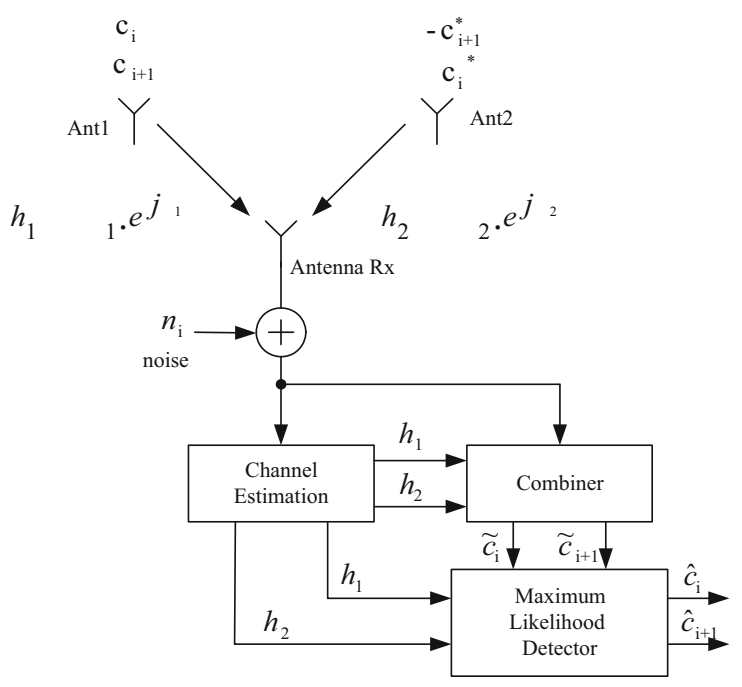

Figure 10. Block diagram of the STC system.

Symbols transmitted by Antenna 1 suffer an attenuation and phase rotation given by

$$
h_{1}=\alpha_{1} e^{j \theta_{1}}
$$

while symbols transmitted by Antenna 2 suffer an attenuation and phase rotation given by

$$
h_{2}=\alpha_{2} e^{j \theta_{2}}
$$

where $\alpha_{1}$ and $\alpha_{2}$ are assumed to be independent random variables with Rayleigh distribution and $\theta_{1}$ and $\theta_{2}$ are assumed to be independent random variables with uniform distribution between $-\pi$ and $\pi$ [13]. These random variables are assumed to be constant over two symbol intervals.
The signals at the input of the receiver in the time instant $t_{i}$ and $t_{i+1}$ are respectively given by

$$
\begin{aligned}
r_{i} & =h_{1} c_{i}-h_{2} c_{i+1}^{*}+n_{i} \\
r_{i+1} & =h_{1} c_{i+1}+h_{2} c_{i}^{*}+n_{i+1}
\end{aligned}
$$

where $n_{i}$ and $n_{i+1}$ are the AWGN noise samples at time instants $t_{i}$ and $t_{i+1}$, respectively. In order to obtain full diversity gain, the detector must have perfect knowledge of the channel parameters. The channel estimation block is responsible for providing these parameters. The combiner uses this information to obtain the diversity provided by the space-time coding, combining the signals as follows:

$$
\begin{aligned}
\hat{c}_{i} & =r_{i} h_{1}^{*}+r_{i+1}^{*} h_{2} \\
& =\left(\alpha_{1}^{2}+\alpha_{2}^{2}\right) c_{i}+n_{i} h_{1}^{*}+n_{i+1}^{*} h_{2} \\
\hat{c}_{i+1} & =h_{1}^{*} r_{i+1}-h_{2} r_{i}^{*} \\
& =\left(\alpha_{1}^{2}+\alpha_{2}^{2}\right) c_{i+1}-n_{i}^{*} h_{2}+n_{i+1} h_{1}^{*}
\end{aligned}
$$

The performance of the Alamouti scheme is equivalent to the performance of the Maximum Ratio Combiner (MRC), except for a penalty of $3 \mathrm{~dB}$ due to the power division in the two transmitting antennas and the double noise addition.

4.3.3. Space-Time Coding and OFDM: There are two different ways to associate the Alamouti scheme with the OFDM transmission technique. The first one uses two OFDM symbols to build the space-time transmission matrix, resulting in a space-time block coding OFDM (STBC-OFDM) scheme [20]. Figure 11 shows the block diagram of this system.

The transmission matrix is given by

\begin{tabular}{c|cc} 
& Ant1 & Ant2 \\
\hline$k^{\text {th }}$ carrier of $i^{\text {th }}$ OFDM symbol & $c_{i}$ & $-c_{i+1}^{*}$ \\
$k^{\text {th }}$ carrier of $(i+1)^{\text {th }}$ OFDM symbol & $c_{i+1}$ & $c_{i}^{*}$
\end{tabular}

Eq. (7) can still be used to obtain a diversity gain from the received signals. The channel frequency response is assumed to be constant over two OFDM symbol periods, which means that the channel coherence time is assumed to be larger than the duration of two OFDM symbols. This scheme does not require a flat frequency response for two or more sub-carriers. Thus, this approach is suitable for 


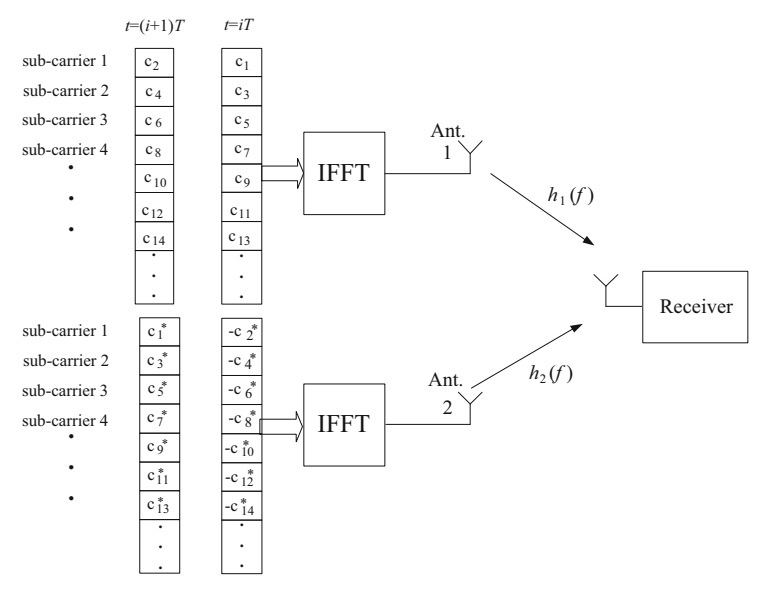

Figure 11. Block diagram of the STBC-OFDM system.

channels that have small coherence bandwidth and large coherence time [17].

The receiver must know the channel frequency response in order to obtain full diversity gain. Pilot subcarriers are introduced in the OFDM symbol to help estimation of the channel frequency response. Figure 12 shows pilot sub-carriers in an STBC-OFDM system.

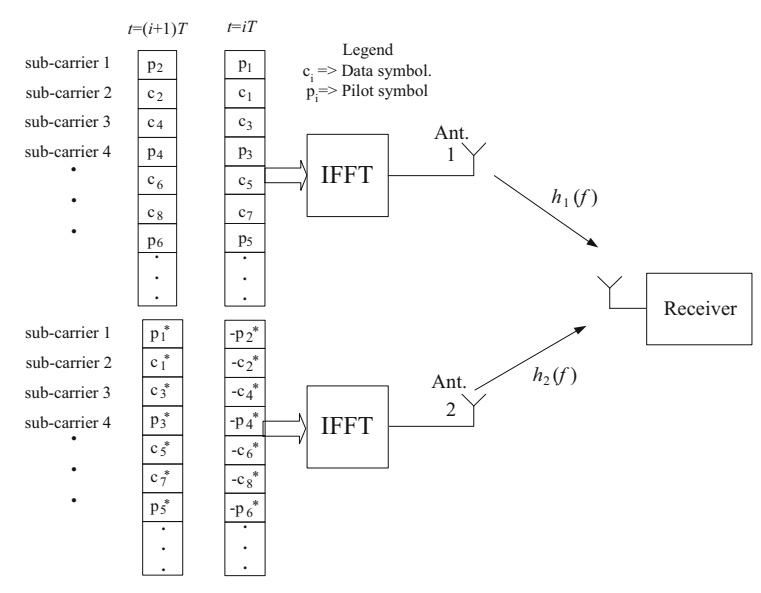

Figure 12. Pilots sub-carriers in an STBC-OFDM system.

The pilot symbols, $p_{i}$, are known to the receiver. Thus, it is possible to estimate the channel frequency response for the pilot sub-carriers. For high sinal-to-noise ratios, the received signals at the $i^{\text {th }}$ pilot sub-carrier frequency, $f_{i}$, are given by

$$
\begin{aligned}
r_{k} & =p_{k} h_{1}\left(f_{i}\right)-p_{k+1}^{*} h_{2}\left(f_{i}\right) \\
r_{k+1} & =p_{k+1} h_{1}\left(f_{i}\right)+p_{k}^{*} h_{2}\left(f_{i}\right)
\end{aligned}
$$

Assuming that $p_{k}=p_{k+1}=p$ and $p \in \Re$, solving (9) for $h_{1}\left(f_{i}\right)$ and $h_{2}\left(f_{i}\right)$ results in

$$
\begin{aligned}
& h_{1}\left(f_{i}\right)=\frac{r_{k+1}+r_{k}}{2 p} \\
& h_{2}\left(f_{i}\right)=\frac{r_{k+1}-r_{k}}{2 p}
\end{aligned}
$$

In order to obtain an estimation of the frequency response for all frequencies, it is necessary to interpolate the estimation obtained in (10). There are several different interpolation techniques that can be used to obtain an estimation of the channel frequency response at the frequencies of the data sub-carriers [18].

The second alternative to combine space-time coding with the OFDM transmission technique is to use two adjacent sub-carriers to build the space-frequency transmission matrix, which results in a space-frequency block coding OFDM (SFBC-OFDM) scheme [21]. Figure 13 presents the block diagram of this system.

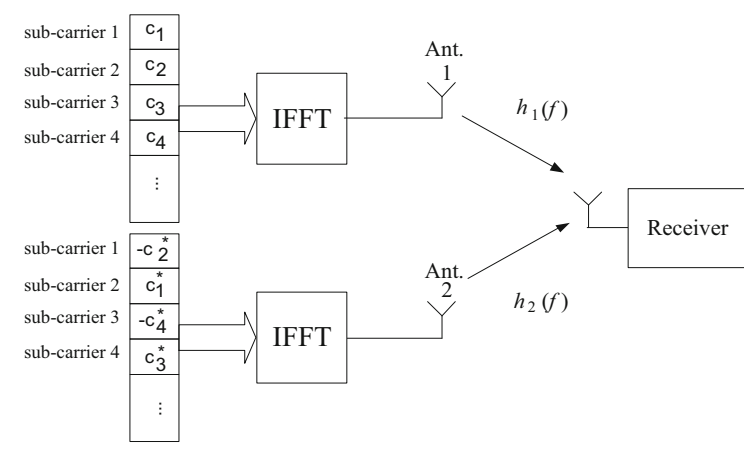

Figure 13. Block diagram of the SFBC-OFDM system.

The transmission matrix is given by

\begin{tabular}{c|cc} 
& Ant1 & Ant2 \\
\hline$i^{\text {th }}$ carrier of $k^{\text {th }}$ OFDM symbol & $c_{i}$ & $-c_{i+1}^{*}$ \\
$(i+1)^{\text {th }}$ carrier of $k^{\text {th }}$ OFDM symbol & $c_{i+1}$ & $c_{i}^{*}$
\end{tabular}

Again, Eq. (7) can be used to obtain the diversity gain from the received signals, where $r_{j}$ is the received signal on the $j^{\text {th }}$ sub-carrier at the same OFDM symbol. The channel frequency response must be the same for two adjacent sub-carriers and time-invariant during one OFDM symbol interval.

It is also possible to use pilot carriers and linear interpolation to estimate the channel frequency response. Here, two adjacent pilot sub-carriers are used to estimate the channel frequency response that must be the same for both sub-carriers.

The received signals at the frequencies of the pilot sub-carriers $f_{i}$ and $f_{i+1}$ are given by

$$
\begin{aligned}
r_{i} & =p_{i} h_{1}\left(f_{i}\right)-p_{i+1}^{*} h_{2}\left(f_{i}\right) \\
r_{i+1} & =p_{i+1} h_{1}\left(f_{i+1}\right)+p_{i}^{*} h_{2}\left(f_{i+1}\right)
\end{aligned}
$$


where $h_{1}\left(f_{i}\right)$ is equal to $h_{1}\left(f_{i+1}\right), p_{i}=p_{i+1}=p \in \Re$ and then (10) is used to obtain an estimation of the channel frequency response on the frequencies of the pilot sub-carriers. The estimation of the channel frequency response at the frequencies of the data sub-carriers can be obtained by using an interpolation algorithm.

Both schemes (STBC-OFDM and SFBC-OFDM) presented here have advantages and disadvantages, which are related to the correlation among these sub-channels. Doppler spread and frequency response of the channel define which scheme should be used. In order to define which scheme is suitable for a DTV Standard, the performance of both approaches were compared for the channels presented in Table 6 .

The mobility of the receiver is simulated by multiplying each path of the channel by a random variable with Rayleigh distribution and mean square value $\sigma_{r}^{2}=1$. The phase of each path is added to a random variable uniformly distributed between $-\pi$ and $\pi$. Two speeds have been considered: $60 \mathrm{~km} / \mathrm{h}$ for channel $13(216 \mathrm{MHz})$ and $120 \mathrm{~km} / \mathrm{h}$ for channel $69(806 \mathrm{MHz})$. The mobility of the receiver results in a time-variant channel. The space-time or space-frequency decoders require that the channel must be constant over the duration of a codeword. It means that the channel must be time-invariant for one OFDM symbol duration for an SFBC-OFDM. For STBC-OFDM, the channel must be time-invariant during at least two OFDM symbol intervals.

Table 11 presents the system parameters used in the simulations and Figure 14 presents the performance of the STBC-OFDM for a receiver moving at $60 \mathrm{~km} / \mathrm{h}$ and channel 13, which results in a Doppler spread of $12 \mathrm{~Hz}$. Figure 15 presents the performance of the SFBC-OFDM for the same conditions.

Table 11. System parameters

\begin{tabular}{|l|c|}
\hline Parameters & Value \\
\hline Data Modulation & QPSK \\
\hline Total Number of sub-carriers & 2048 \\
\hline Sub-carrier spacing & $3.97 \mathrm{kHz}$ \\
\hline Pilot modulation & $\mathrm{BPSK}$ \\
\hline Guard-time interval & $\mathrm{T} / 16$ \\
\hline Total OFDM symbol duration & $267.5 \mu \mathrm{s}$ \\
\hline Equalization & Perfect estimation \\
\hline
\end{tabular}

Comparing Figures 14 and 15 it is possible to conclude that the STBC-OFDM scheme is better than the SFBC-OFDM scheme in all channels. The performance of SFBC-OFDM is highly penalized when the coherence bandwidth of the channel is reduced. The reduction of the coherence bandwidth does not severely affect the STBCOFDM scheme because this scheme does not require the same frequency response for two adjacent sub-carries, as

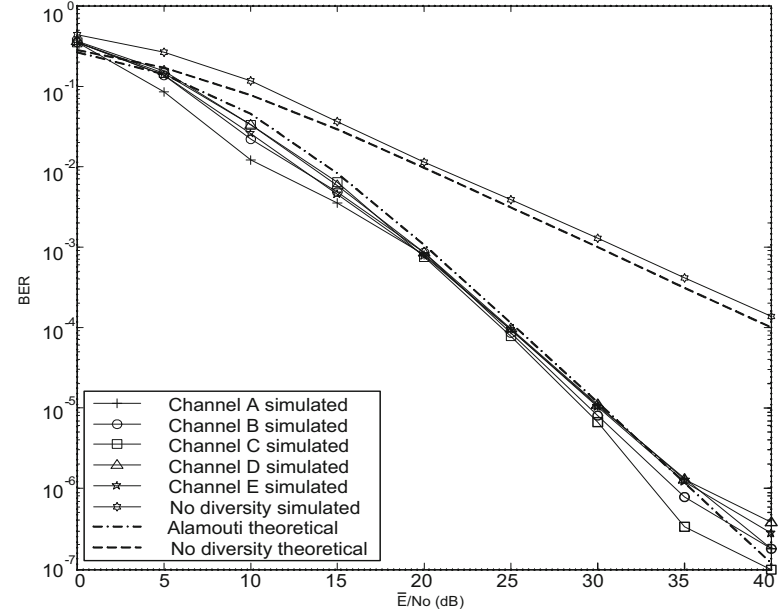

Figure 14. Performance of STBC-OFDM system with Doppler spread of $12 \mathrm{~Hz}$.

the SFBC-OFDM does.

Figure 16 presents the performance of the STBCOFDM for a receiver moving at $120 \mathrm{~km} / \mathrm{h}$ and channel 69 , which results in a Doppler spread of $89 \mathrm{~Hz}$. Figure 17 presents the performance of the SFBC-OFDM for the same conditions.

Comparing Figures 16 and 17 it is possible to conclude that the SFBC-OFDM is better than SFBC-OFDM when the mobility of the receiver is high. These behaviors are related to the fact that the frequency selectivity of the channels plays a minor role in the performance of the schemes, while the Doppler spread plays a major role. The fact that SFBC-OFDM requires a lower channel coherence time than STBC-OFDM results in its better performance.

The MI-SBTVD design team has decided to use STBC-OFDM, because the most common scenario for DTV reception is with a small Doppler spread. The simulation results presented in this paper show that the performance of the STBC-OFDM in this case is better than the performance of the SFBC-OFDM for all channel profiles.

\subsection{OFDM FRAME STRUCTURE}

The structure of the OFDM frame is based on the ISDB-T standard. There are three available modes: Mode 1, employing a 2048-point FFT, Mode 2 with 4096-point FFT and Mode 3 with 8192-point FFT. The first mode is robust to Doppler spread but it is not suitable for Single Frequency Network (SFN) [19]. Mode 3 is suitable for SFN, but it is not robust to Doppler spread. Mode 2 is an intermediate solution that can be used in low-speed mobile reception in an SFN.

The OFDM symbol is composed by 13 segments. The number of sub-carriers per segment depends on the FFT 


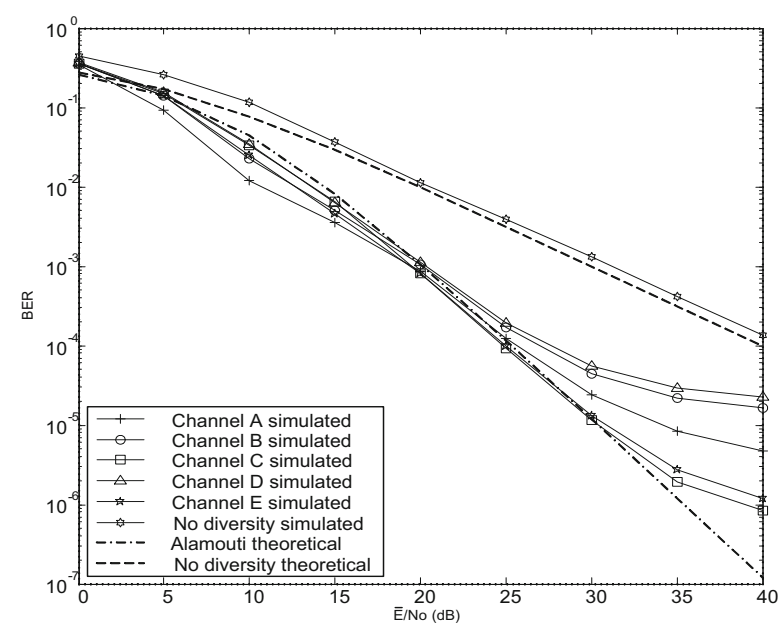

Figure 15. Performance of SFBC-OFDM system with Doppler spread of $12 \mathrm{~Hz}$.

length. These segments can be freely grouped to transmit up to three different data streams (layers). A segment is composed by data carriers, scattered pilots carriers (SP), transmission and multiplexing configuration and control carriers (TMCC). There are also auxiliary carriers (AC) that can be used as complementary signaling. MI-SBTVD uses these carriers to identify the first OFDM symbol of the Space Time Code. Table 12 shows the composition of a segment based on the FFT length.

Table 12. Number of carriers per segment.

\begin{tabular}{|l|c|c|c|}
\hline & $\begin{array}{c}\text { Mode 1 } \\
\text { 2048 FFT }\end{array}$ & $\begin{array}{c}\text { Mode 2 } \\
\text { 4096 FFT }\end{array}$ & $\begin{array}{c}\text { Mode 3 } \\
8192 \text { FFT }\end{array}$ \\
\hline Total & 108 & 216 & 432 \\
\hline Data & 96 & 192 & 384 \\
\hline SP & 8 & 18 & 36 \\
\hline TMCC & 1 & 2 & 4 \\
\hline AC & 2 & 4 & 8 \\
\hline
\end{tabular}

The OFDM frame is composed by 204 OFDM symbols. This number guarantees an integer number of Reed Solomon codewords, regardless the code rate, guard time interval, modulation order, segment combination or FFT length. The number of LDPC codewords is a multiple of 0.5 , which means that it may be necessary two OFDM frames to obtain the LDPC synchronism. Table 13 presents the number of codewords per segment, as a function of the modulation order and FFT length.

\section{Performance Analysis}

In this section we present performance results of the modulation and channel coding schemes developed for

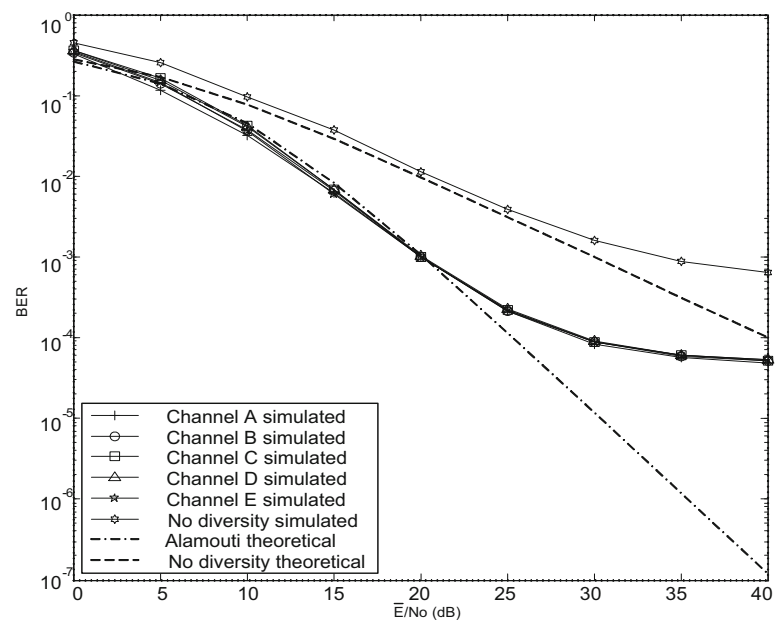

Figure 16. Performance of STBC-OFDM system with Doppler spread of $89 \mathrm{~Hz}$.

Table 13. Number of codewords per segment.

\begin{tabular}{|l|c|c|c|}
\hline Mode & Modulation & LDPC & R\&S \\
\hline \multirow{3}{*}{ Mode 1 } & QPSK & 4.5 & 216 \\
\cline { 2 - 4 } & 16QAM & 9 & 432 \\
\cline { 2 - 4 } & 64QAM & 13.5 & 648 \\
\hline \multirow{3}{*}{ Mode 2 } & QPSK & 9 & 432 \\
\cline { 2 - 4 } & 16QAM & 18 & 864 \\
\cline { 2 - 4 } & 64QAM & 27 & 1296 \\
\hline \multirow{3}{*}{ Mode 3 } & QPSK & 18 & 864 \\
\cline { 2 - 4 } & 16QAM & 36 & 1728 \\
\cline { 2 - 4 } & 64QAM & 54 & 2592 \\
\hline
\end{tabular}

the MI-SBTVD system. All the results have been obtained by computer simulation, using Matlab Simulink. The results presented here are a subset of the results reported to the Brazilian Communications Ministry [22].

As described in Section 3, ITU suggests some typical channel profiles that can be used to test DTV systems with fixed reception (Brazil A, B, C, D and E) and mobile reception (Typical Urban GSM). The performance of the system has been tested for all of these channels, for AWGN channel with and without impulsive noise.

The following general specifications have been considered in the simulations:

- Channel Coding: in the final specification of the system the internal code is a LDPC with block length equal to 9 kbits. In our preliminary tests, we have used LDPC with block length equal to 9 kbits or 39 kbits. The results reported here, when not specified, correspond to LDPC block length equal to $39 \mathrm{kbits}$.

- OFDM Modulation: the simulations were performed using a $2 \mathrm{k}$ OFDM scheme. 


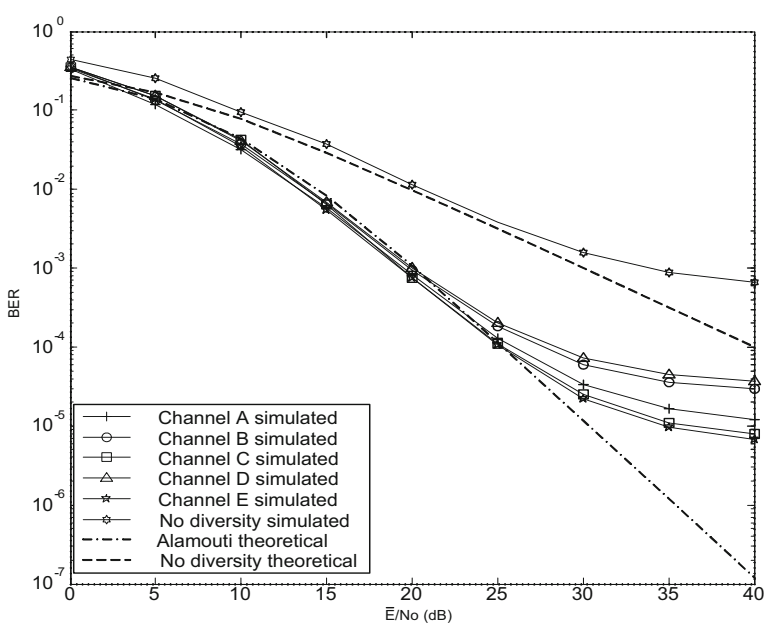

Figure 17. Performance of SFBC-OFDM system with Doppler spread of $89 \mathrm{~Hz}$.

- Guard Time: the guard time was fixed at $1 / 16$ of the OFDM symbol period in all the simulations.

- Channel Estimation and Synchronization: perfect channel knowledge and synchronism are assumed. This choice has been made in order to evaluate the system potential, without regarding to the limitations of one receiver implementation or another.

- Simulation Stopping Criterion: Simulations with a fixed receiver were stopped after the transmission of 39168000 bits (or 1000 LDPC blocks) or after the occurrence of 40000 bit-errors after the RS decoder, whatever comes first. On mobile reception conditions, 3000 LDPC blocks were used for Doppler deviation equal to $119 \mathrm{~Hz}$ and 5000 LDPC blocks were used for Doppler deviation equal to $12 \mathrm{~Hz}$.

- $C / N_{\text {thres }}$ : The carrier-to-noise ratio $C / N$ threshold is defined as the minimum ratio at which no errors are observed at the output of the RS decoder during the simulation period described above (on the graphs, the threshold can be identified by the vertical descent of the bit-error rate). As the simulation process for $C / N$ greater than the threshold is a very long task, with prohibitive simulation time, we have investigated the bit error rate in this condition only in the scenario using 64-QAM modulation, LDPC code with rate equal to $3 / 4$ and for the AWGN channel. In this case, we can not find any bit error after 120 millions of transmitted bits, guaranteeing a bit error rate lower than or equal to $2.9 \times 10^{-8}$, with a $95 \%$ confidence interval.

\subsection{Performance on AWgn Channel}

In order to evaluate the performance on AWGN channel, QPSK and 16-QAM modulation schemes were used with LDPC code rates $1 / 2$ and $7 / 8$, and 16-QAM and 64QAM were used with LDPC code rates of $1 / 2$ and 3/4. Figure 18 shows the results obtained. It can be seen that the $C / N$ threshold is $15.4 \mathrm{~dB}$ when a 64-QAM modulation is used with a LDPC code with rate $3 / 4$. This configuration results in a $19.33 \mathrm{Mbps}$ throughput. If a very robust configuration is needed, a QPSK modulation with a LDPC code with rate $1 / 2$ can be used. In this case, the $C / N$ threshold is equal to $1.3 \mathrm{~dB}$.

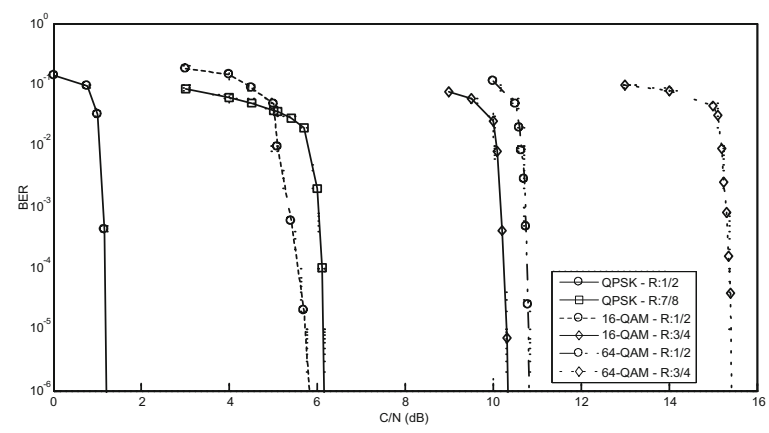

Figure 18. Performance on AWGN channel.

Table 14 summarizes the throughput and $\mathrm{C} / \mathrm{N}$ threshold obtained for each simulated scenario in AWGN channels. It can be seen that the scenario with 16-QAM modulation and LDPC code rate 3/4 has the same throughput of the scenario with 64-QAM and code rate $1 / 2$, but it has lower $C / N$ threshold and, thus, better performance.

In order to investigate the influence of the LDPC block length codes, Figure 19 compares the performance of the system considering 64-QAM, 16-QAM and QPSK modulations and LDPC with lengths equal to $9 \mathrm{k}$ and $39 \mathrm{k}$ with rates equal to $\frac{1}{2}$ and $\frac{3}{4}$. It can be seen that the performance is only $0.2 \mathrm{~dB}$ better if a $39 \mathrm{k}$ LDPC code is used, instead of the 9k LDPC code specified for the MI-SBTVD system.

Figure 20 compares the performance of the proposed system with the results presented in [23] for the ATSC standard. It can be observed that both systems have similar performances. However, it is important to notice that our results have been obtained by simulation and that the results presented in [23] have been obtained from measurements. Thus, we can expect that the performance of the ATSC would be better than the performance of our system in AWGN channel. Actually, the ATSC has the best performance in this kind of channel.

\subsection{Performance ON Brazil-A Through}


Table 14. Summary of throughput and C/N threshold for AWGN channels.

\begin{tabular}{|l|c|c|c|c|c|c|}
\hline & QPSK & QPSK & 16QAM & 16QAM & 64QAM & 64QAM \\
& $1 / 2$ & $7 / 8$ & $1 / 2$ & $7 / 8$ & $1 / 2$ & $3 / 4$ \\
\hline Throughput [Mbps] & 4.23 & 7.52 & 8.59 & 12.89 & 12.89 & 19.33 \\
\hline C/NN $_{\text {threshold }}[\mathrm{dB}]$ & 1.3 & 6.2 & 6.6 & 10.4 & 10.8 & 15.4 \\
\hline
\end{tabular}

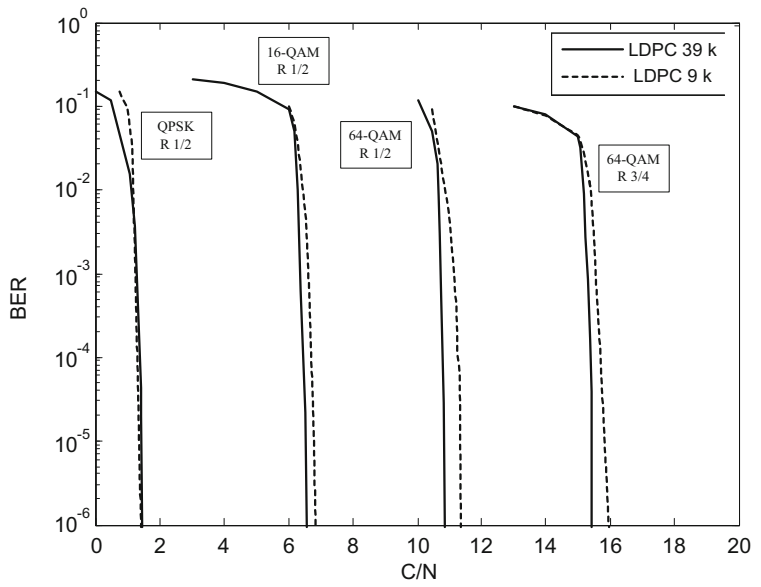

Figure 19. Comparing the influence of the LDPC block lengths.

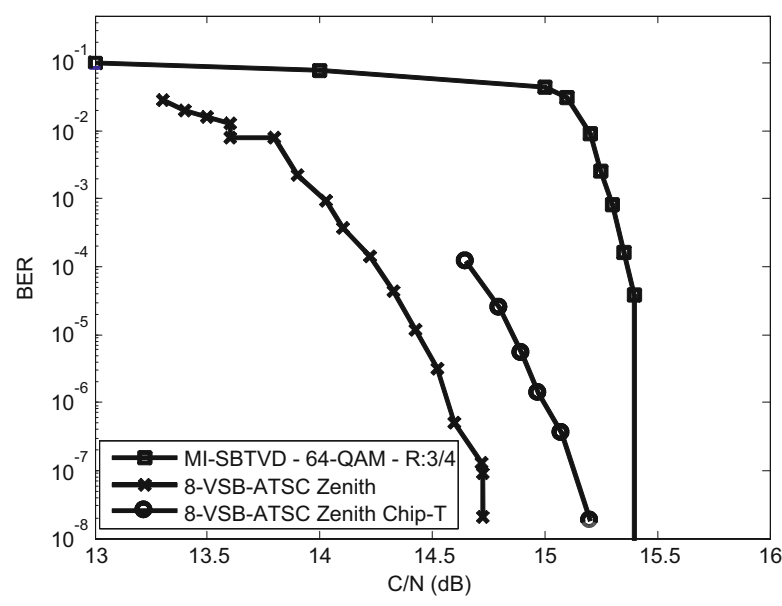

Figure 20. Comparing the performance between MI-SBTVD and ATSC in AWGN channel.

comparisons consider 64-QAM modulation and LDPC code rate $3 / 4$ for the MI-SBTVD system. One can see that the proposed system performs better than ISDB-T and DVB-T standards. The difference in the performances varies from $6 \mathrm{~dB}$ to $19 \mathrm{~dB}$. Again, it is important to notice that the performance of the proposed system has been obtained by simulation and the results presented in [23] for ISDB-T and DVB-T have been obtained from measurements.

\subsection{Performance on ChanNels With IMPUl- SIVE NOISE}

The basic test procedure for channels with impulsive noise was to find, for each value of carrier-to-interference ratio $(C / I)$, the minimum $C / N$ ratio that results in a BER of at most $3 \times 10^{-6}$ (Threshold of Visibility - TOV). As described above, two channel models have been considered: one associated with systems with internal reception (noises generated by the power-supply network, or by direct induction on the receiver), named IMPULI, and another associated with systems with external reception (noises captured by the external antenna, such as the noises generated by the ignition of automobiles), named IMPUL-O. Figure 27 shows the results obtained for the IMPUL-I noise model, for 64-QAM modulation and code-rate $1 / 2$. It can be seen that the proposed system is able to operate with BER below TOV for $C / I$ ratios 


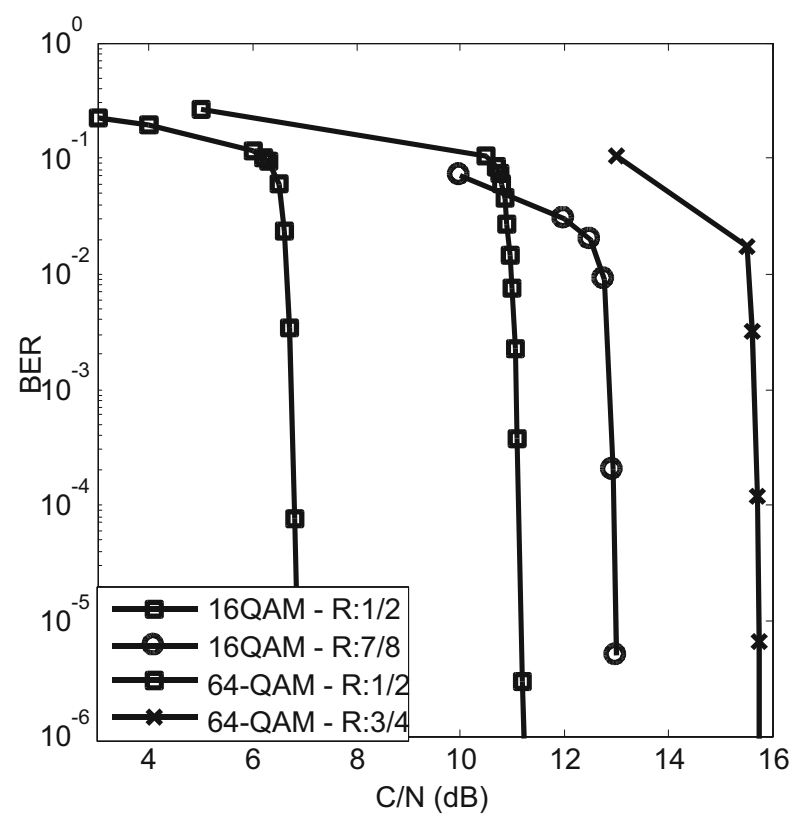

Figure 21. System Performance for Brazil-A Channel.

greater than or equal to $26.5 \mathrm{~dB}$, approximately.

Figure 28 shows the performance of MI-SBTVD under the presence of impulsive noise considering the channel IMPUL-O, for 64-QAM modulation and LDPC code with rate 3/4, which presents throughput greater than 19 Mbps. It can be seen that the proposed system is able to operate with BER smaller than TOV for $\mathrm{C} / \mathrm{I}$ ratios greater than or equal to $33.5 \mathrm{~dB}$, approximately.

\subsection{Influence of THE Alamouti's SCHEMe in The System Performance}

To evaluate the efficiency of the Alamouti space-time coding scheme, the $2 \times 1$ mode was compared to the $1 \times 1$ mode using 64-QAM with rate 3/4 for Brazil-A through Brazil-E channels. An example of the obtained results is presented in Figure 29 for the Brazil-A channel. The performance improvement obtained with the use of two antennas was substantial, with gains ranging from $1.9 \mathrm{~dB}$, for Brazil-C channel, up to $4 \mathrm{~dB}$ for Brazil-B channel.

\subsection{Mobile Reception Performance}

The channel model that represents mobile reception is typical urban GSM. Two mobility scenarios have been considered: one with low speed (around 25 kilometers per hour for the UHF channel 14) and another one with high speed (around 130 kilometers per hour for the UHF channel 14). In all figures presented in this sub-section,the value of the $C / N$ threshold is the last point on the curve. The vertical line down from that point indicates that it was

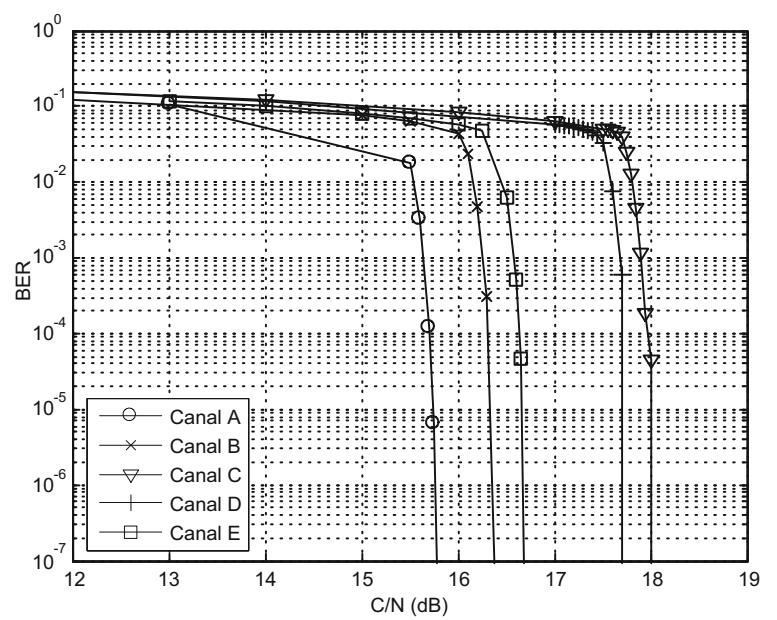

Figure 22. System Performance for Brazil-A through Brazil-E channels using 64-QAM and LDPC code rate 3/4.

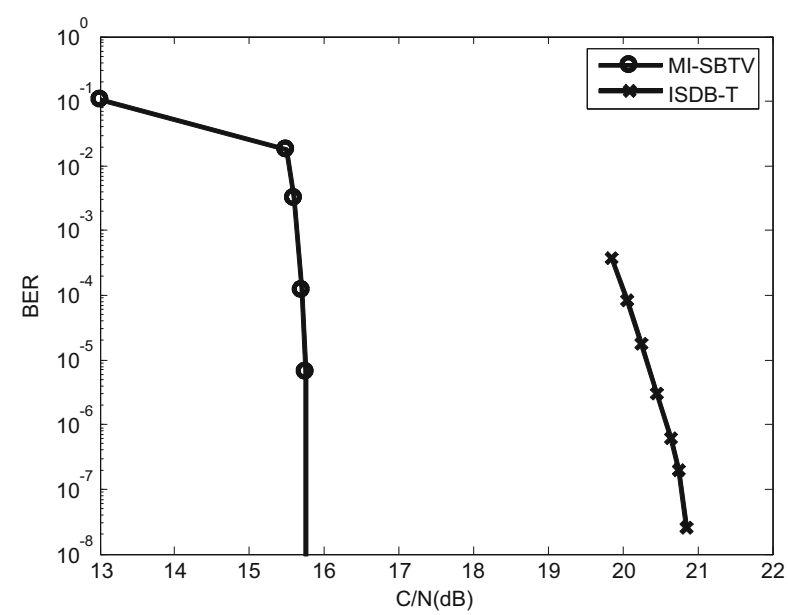

Figure 23. Comparing the performance between MI-SBTVD and ISDB-T for Brazil-A Channel.

not possible to observe any bit errors beyond the threshold during the total simulation interval (corresponding to 120 and 200 million transmitted bits for Doppler deviations of $12 \mathrm{~Hz}$ and $60 \mathrm{~Hz}$, respectively). Figures 30 and 31 show the system performance for 16-QAM modulation with code rate $1 / 2$ and Doppler deviations $12 \mathrm{~Hz}$ and $60 \mathrm{~Hz}$, respectively, while Figures 32 and 33 illustrate the performance for QPSK modulation, code rate $1 / 2$, and Doppler deviations equal to $12 \mathrm{~Hz}$ and $60 \mathrm{~Hz}$, respectively. These figures also show the effect of the correlation between the received signals (coming from the two transmitting antennas) on the system performance. One can observe that the system performance gets worse as the correlation between the received signals increases. Based on the results 


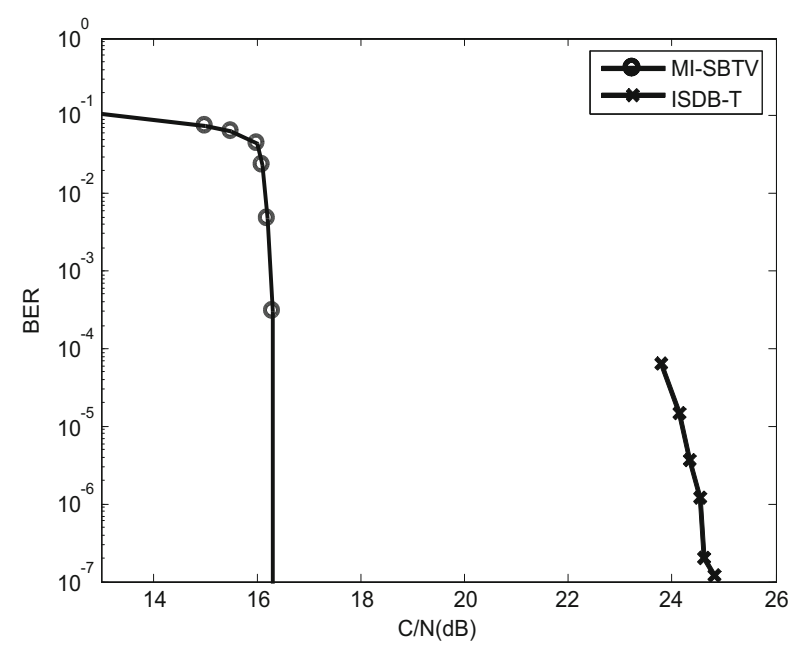

Figure 24. Comparing the performance between MI-SBTVD and ISDB-T for Brazil-B Channel.

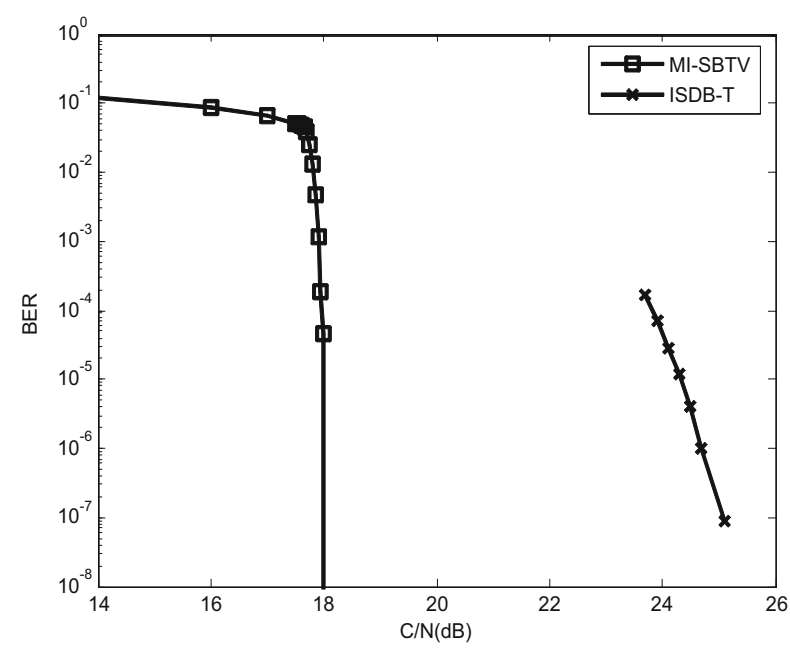

Figure 25. Comparing the performance between MI-SBTVD and ISDB-T for Brazil-C Channel.

presented here, it can be concluded that the system is able to operate with high efficiency for mobile reception using more robust modulation schemes, such as QPSK and 16QAM. Figure 34 compares the performance of the system with two transmitting antennas when one of the antennas is turned off (without any change at the receiver). The configuration with QPSK modulation, code rate $1 / 2$ and Doppler deviation of $60 \mathrm{~Hz}$ was used. Similar results to the ones shown in Figure 34 were obtained for 16-QAM modulation, code rate equal to $1 / 2$, and Doppler deviation $12 \mathrm{~Hz}$. From these results, it is possible to observe a drop of 5-9 dB on the system performance when one of the transmitting antennas is turned off.

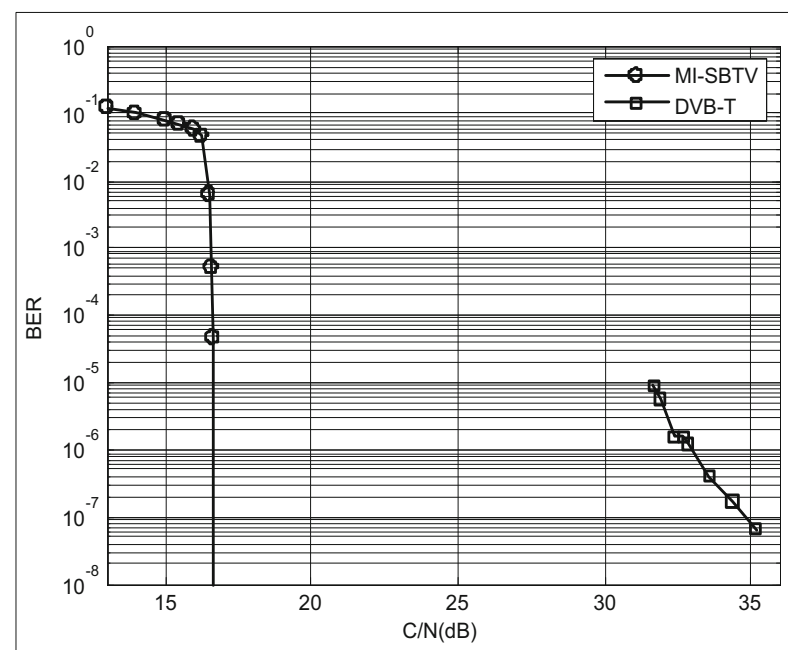

Figure 26. Comparing the performance between MI-SBTVD and DVB-T for Brazil-E Channel.

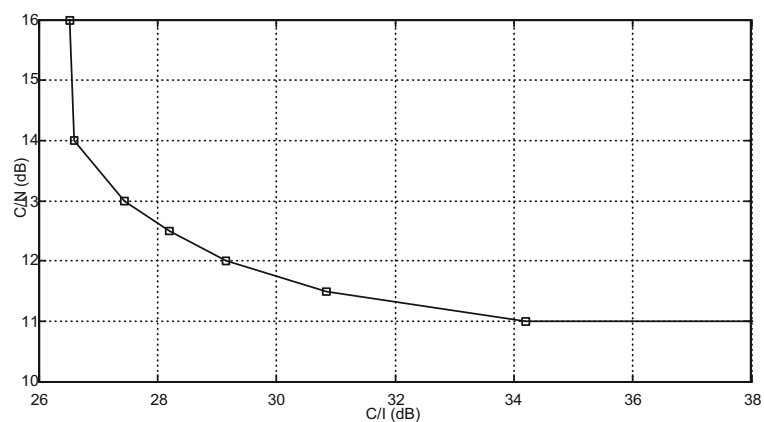

Figure 27. Performance with impulsive noise and internal reception.

\section{IMPLEMENTATION ASPECTS}

This section introduces some implementation techniques which were chosen to exemplify what kind of algorithms engineers have to deal with when designing a physical layer system for ASIC or FPGA, particularly when the target application is digital television. Although the algorithms are presented here using a systemic approach, they can also encompass behavioral abstractions, including loop controls and processes. The behavioral approach is typically used for synchronous operations such as filtering or any other digital signal processing algorithm.

An important aspect in the design of algorithms for FPGA is that there can be sub-systems running with different clock rates. This frequently occurs when an interface, such as Ethernet or DVB-SPI, needs to be implemented for communication with other devices. It also occurs when exploiting flexibilities between parallel and serial architectures, aiming at increasing processing ca- 


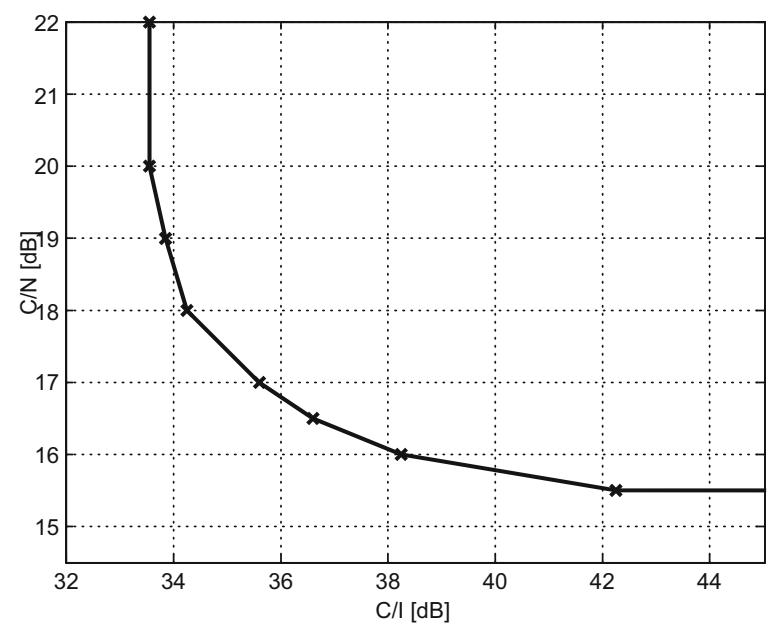

Figure 28. Performance with impulsive noise and external reception.

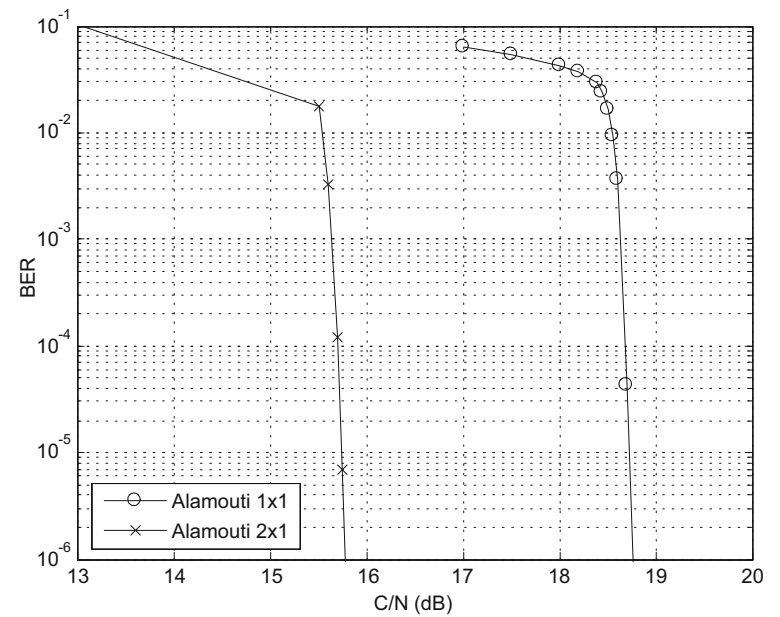

Figure 29. Comparing the performance between Alamouti $1 \times 1$ and 2 $\mathrm{x} 1$ over Brazil-A channel.

pacity. Perhaps the greatest challenge in the design of new algorithms for an FPGA is the trade-off between high speed processing and silicon area occupation.

For example, when a Cordic division algorithm, which is very expensive in logical resources (silicon area), needs to perform $N$ divisions in a given time slot, it may not have enough hardware resources to implement all the $N$ operations in parallel. In this case, some operations have to be serialized, increasing the clock, to implement the division algorithm in a semi-sequential or totally sequential mode.

Another example is the implementation of an LDPC decoder using an eIRA code for rate $3 / 4$ and codeword

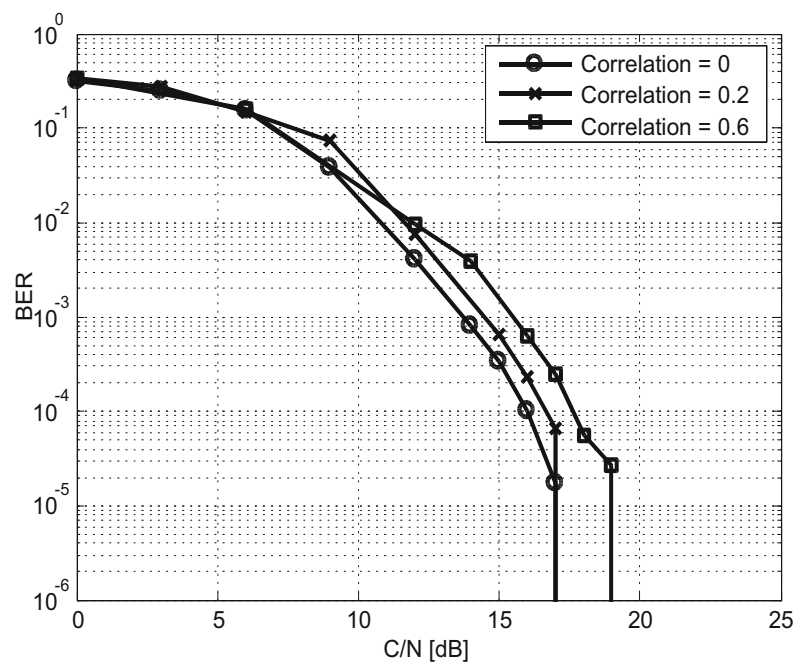

Figure 30. Mobile reception with $16 \mathrm{QAM}, \mathrm{R}=1 / 2$ and Doppler deviation equal to $12 \mathrm{~Hz}$.

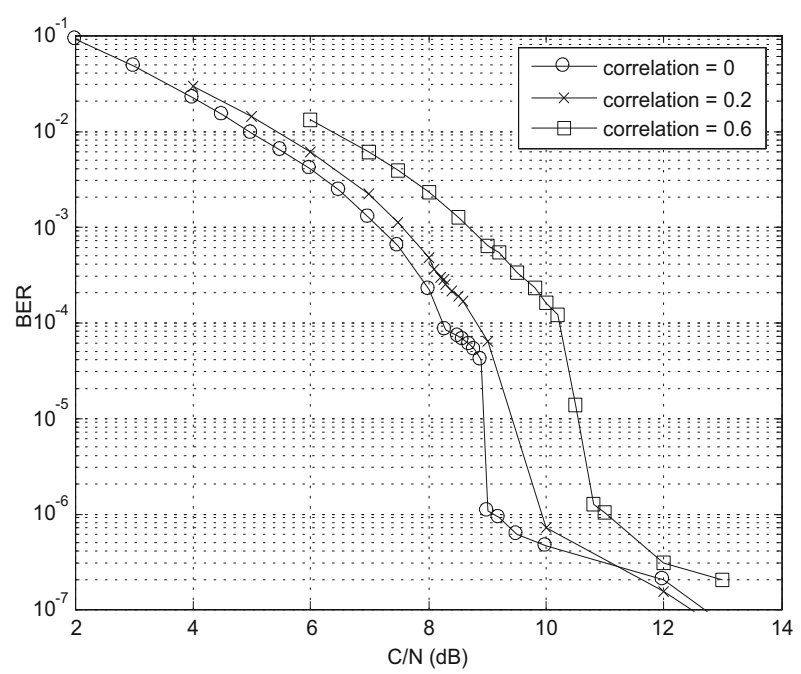

Figure 31. Mobile reception with 16QAM, $\mathrm{R}=1 / 2$ and Doppler deviation equal to $60 \mathrm{~Hz}$.

length of 9792 bits [26]. The implementation of such a decoding algorithm in an FPGA Xilinx Virtex 4 SX35 is not feasible with a full parallel architecture. For this reason, a semi-sequential approach, in the form of a trellis structure, had to be used with a parallelization degree of 51 , a number just sufficient to fit the algorithm in the device.

In the remainder of this section two algorithms to exemplify the implementation developed for the MISBTVD project are described. A digital down-converter and its advantages over its analog version are first presented. We then describe the rate control algorithm which 


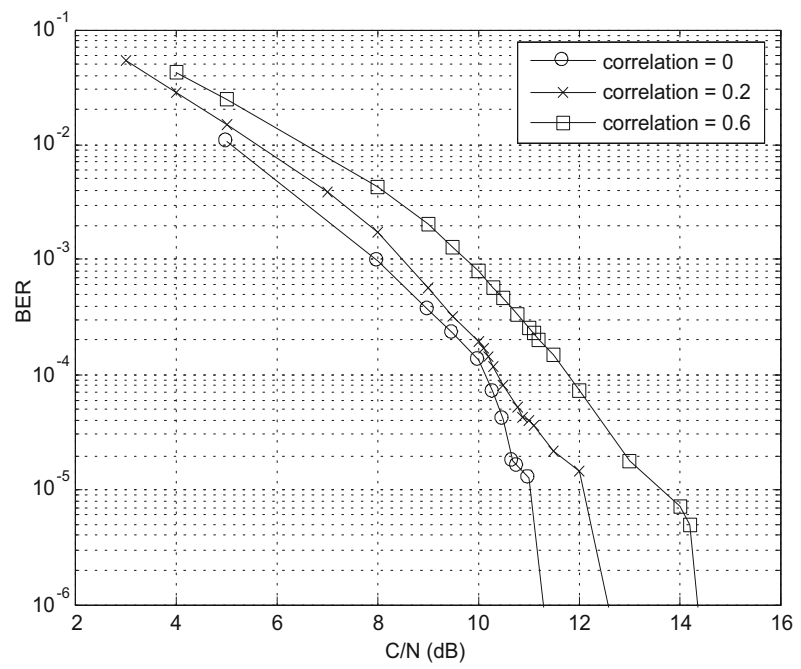

Figure 32. Mobile reception with QPSK, $\mathrm{R}=1 / 2$ and Doppler deviation equal to $12 \mathrm{~Hz}$.

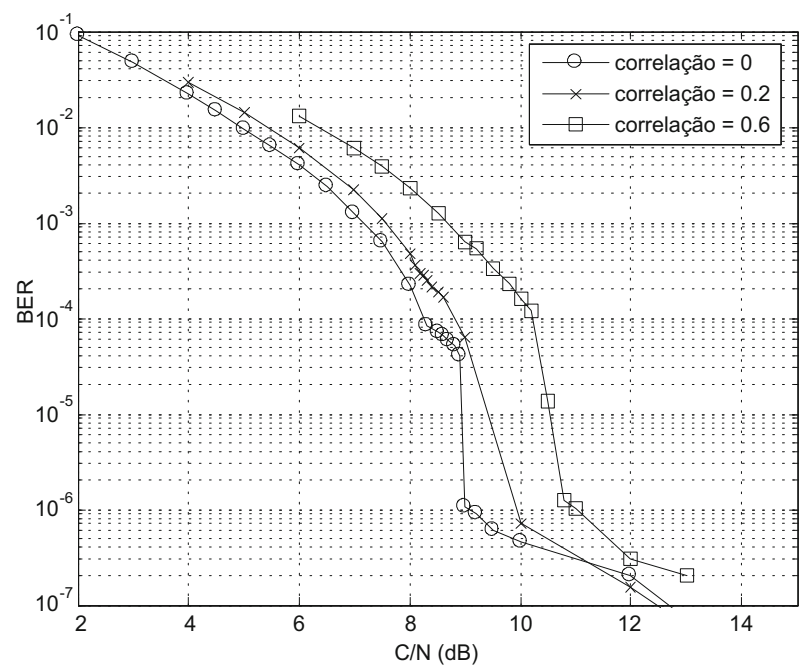

Figure 33. Mobile reception with QPSK, $R=1 / 2$ and Doppler deviation equal to $60 \mathrm{~Hz}$.

is responsible for integrating the video generation, the channel encoding and the modulation sub-systems in the transmitter prototype.

\subsection{Digital Down-Converter}

The receiver design was based on a software defined radio (SDR) architecture and consists basically of a superheterodyne receiver, with the IF stage implemented digitally in software, as shown in Figure 35. In a superheterodyne scheme, the receiver front-end is typically split into an RF and an IF stage, as shown in Figure 36. The RF stage is sensitive to a wide frequency range, in

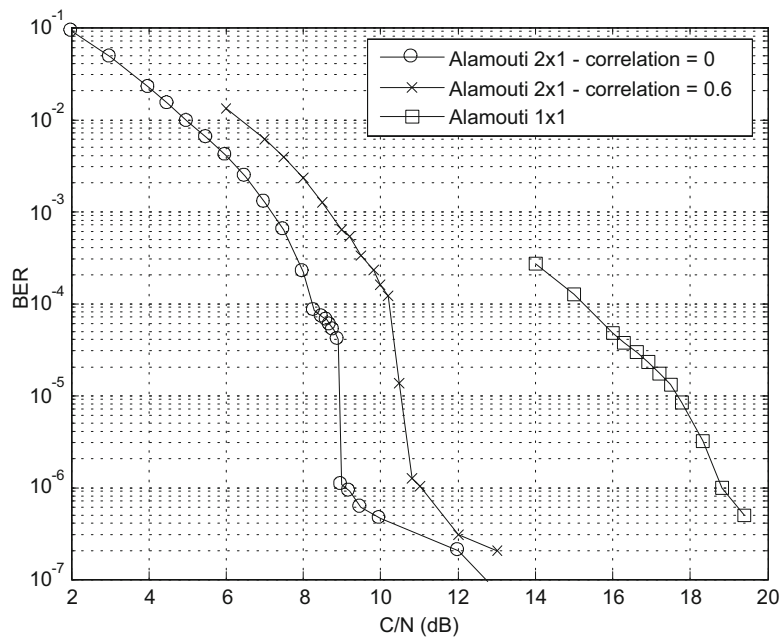

Figure 34. Mobile reception with QPSK, $\mathrm{R}=1 / 2$, Doppler deviation equal to $60 \mathrm{~Hz}$ and Alamouti 1x1 and 2x1.

our case $30 \mathrm{MHz}$, and may have one or two tuned stages which are necessary to allow television viewers to select the desired channel. After a channel is selected by adjusting the local oscillator frequency, the incoming signal is multiplied by the oscillator signal in a mixer, which results in down-converting this incoming signal to an intermediate frequency (IF). In the IF stage, the signal is filtered to extract the desired channel, allowing the rest of the circuitry to be sensitive to a shorter frequency range, in our case $6 \mathrm{MHz}$. Finally, the resulting signal is downconverted to baseband and can be demodulated by software.

There are many advantages in the use of a SDR receiver compared to a traditional super-heterodyne architecture, such as:

- The digital down-converter eliminates the drawbacks of phase and gain mismatch associated with its analog version. The local oscillator, used in the analog down-conversion, is replaced by a simple lookup table containing samples of a sinusoidal wave, as shown in Figure 37. All operations are digitally implemented, which avoid unbalancing the in-phase (I) and quadrature-phase $(\mathrm{Q})$ baseband signals.

- Channelization can be implemented in software by digital low-pass filtering. The analog version of this process demands for analog filters with very stringent requirements, which are much more difficult to implement compared to their digital versions.

Prior to digital down-conversion to obtain I and Q baseband signals, it is necessary to digitize the analog signal centered at the intermediate frequency IF. The first 


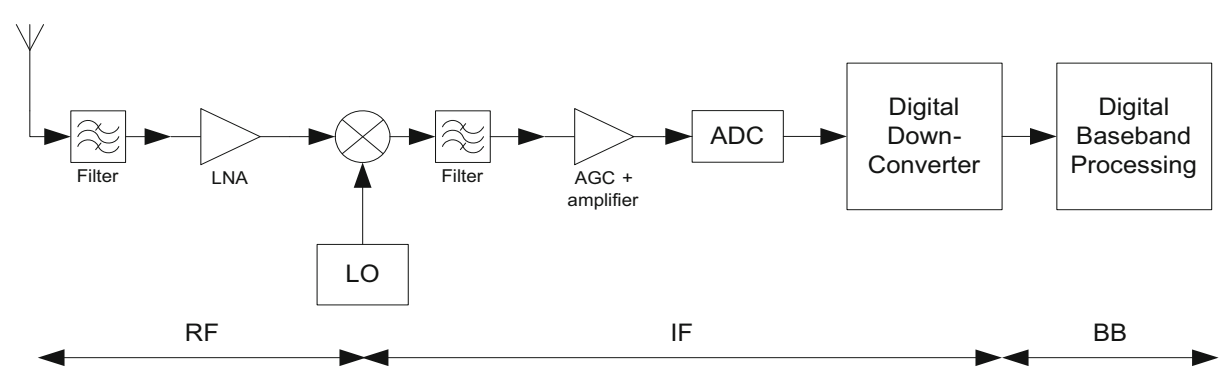

Figure 35. Software-defined radio architecture.

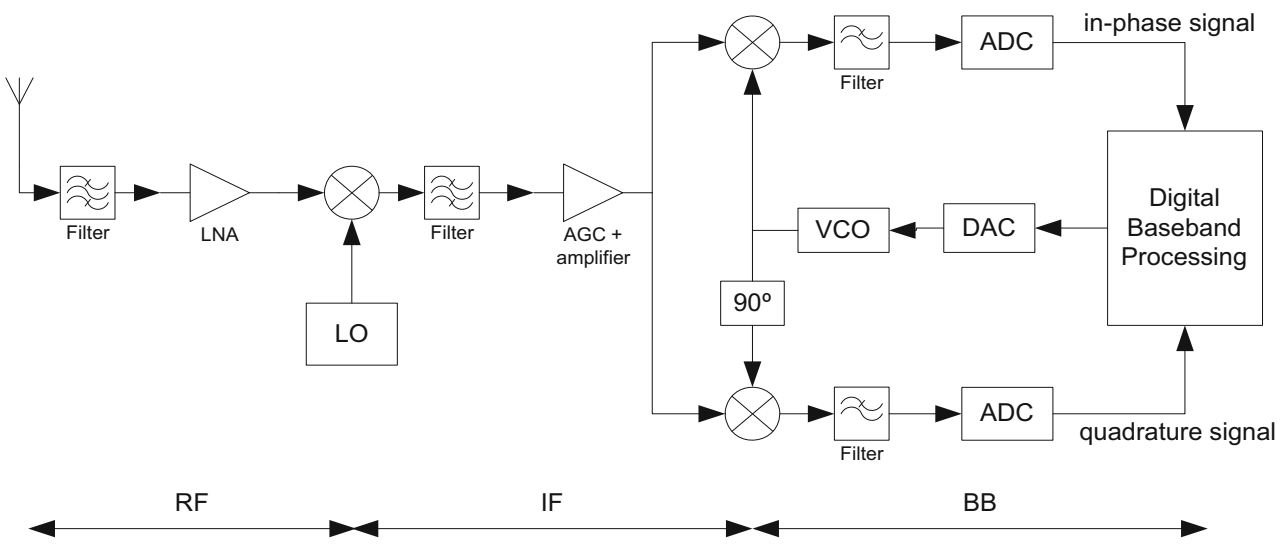

Figure 36. Traditional super-heterodyne receiver.

prototype of the MI-SBTVD system was chosen to operate with an IF of $8.1270 \mathrm{MHz}$, which corresponds exactly to the same sample frequency $f_{s}$ used for I and Q baseband signals. The reason for that choice is that, when the analog signal is sampled with $4 f_{s}$, the digital mixing has a very simple structure, as shown in Figure 38, which replaces the look-up tables and the two multiplications by a simple switch and a "negate" operation (times -1). If the intermediate frequency is $f_{s}$, then it is necessary to mix the sampled IF signal with two quadrature sinusoidal waves of frequency $f_{s}$, in order to recover the baseband signals. These sinusoidal waves need to be sampled at the same rate $4 f_{s}$ as the input signal, which results in two periodic sequences: $1,0,-1,0$ for the cosine wave and $0,1,0,-1$ for the sine wave. The mixing operation is performed by multiplying the IF signal with these two periodic sequences, $1,0,-1,0$ and $0,1,0,-1$, in the I and $\mathrm{Q}$ branches, respectively. To save computational effort, a switch is used in each branch, splitting the samples multiplied by 1 and -1 and the samples multiplied by 0 . Subsequently, a low pass filter with a polyphase structure is used to decimate the mixed signal, resulting in the I and $\mathrm{Q}$ signals at baseband sampling rate $f_{s}$. The advantage of using polyphase decimators is that the filtering operation is performed on the lower baseband frequency $f_{s}$, which also saves computational effort [24] [25].

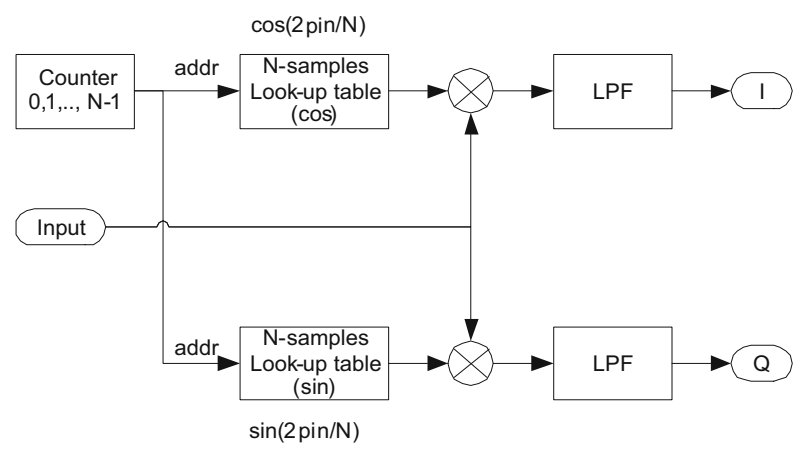

Figure 37. A digital down-converter implementation using look-up tables.

6.2. RATE CONTROL FOR THE TRANSMission 


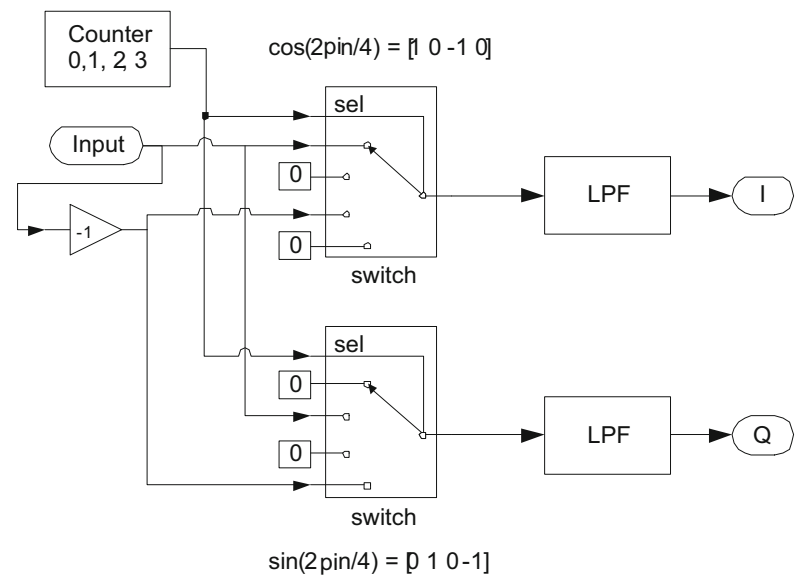

Figure 38. A low-complexity implementation of a digital down-converter scheme.

\section{SYSTEM}

The main idea for rate control is to transmit null data packets, that is, packets which do not have useful information, always when new data is required by advanced stages and that required data is still not available to be processed. This occurs, for example, when the video data rate to be transmitted is below the system throughput, which forces the system to fulfill the difference with some dummy information, in this case the null packets, which can be identified and later removed at the receiver. We, therefore, have two types of packets which can be transmitted. Besides, once the transmission of one packet type has started, it is necessary to complete that transmission before switching to another packet type. Although this rule might sound obvious, it is very important not to mix the two packet types together, otherwise the null ones will not be recognized by the receiver and consequently they cannot be removed. Obviously, this will corrupt the video data, since the receiver will not be able to restore the original video stream.

In this way, two states can be defined for the rate control algorithm: "read data packet" and "read null packet". These states are imbedded in the register "Reading State", which stays in the same state at least during 204 valid consecutive bytes, which is the packet size for both packet types. The purpose of this register is to inform if there is an entire data packet available for reading, and its content is always updated whenever the last byte of one packet is read by the system. Therefore, whenever a new packet is about to be read, that is, when the "frame index" reaches the value 203, the control signal "Data Packet Available" is inspected and saved in the register "Reading State".

With these concepts in mind, it is easier to understand the control signals of the algorithm, as shown in Figure 39. The "Reading State" controls the selectors "Data
Selector" and "Valid Selector", which drive the outputs "Byte Output" and "Valid Output", respectively. When the state is " 0 ", that is, when there is no data available for reading, then a null packet is read through a ROM memory. The reading address is provided by the counter "frame index" and the signal "Valid" is a delayed version for the reading request coming from the LDPC encoder.

On the other hand, when the "Reading State" is " 1 ", it means that there is at least one data packet available for reading, and then the signal "Reading Request", coming from the LDPC encoder, is passed as a read enable control to the input FIFO (First In First Out) which records the data packets. This FIFO is not shown in Figure 39, but in Figure 40. As a result, the bytes read from this FIFO are made available on the input ports "Input Byte" and "Input Valid". The latter signal "Input Valid" is redundant here because it is always " 1 " when the "Reading Request" is " 1 ", and " 0 " otherwise.

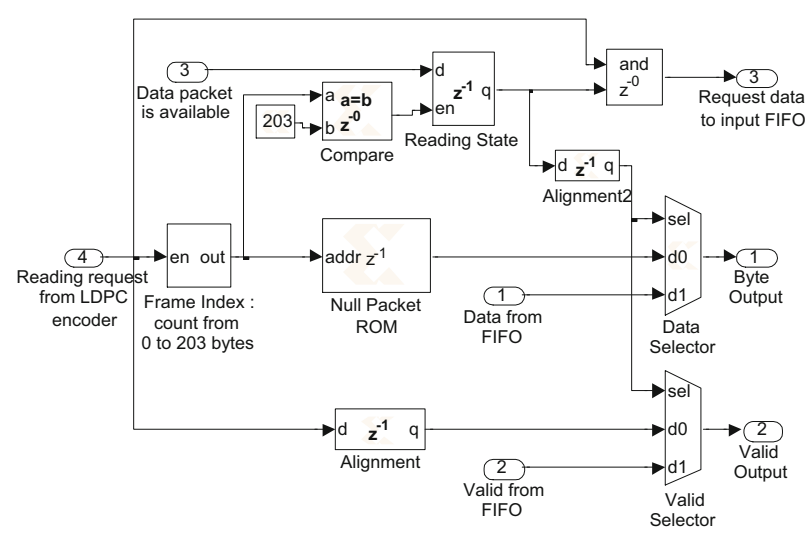

Figure 39. Block diagram showing the implementation of the rate control system. This block diagram is implemented using Xilinx System Generator for Matlab/Simulink.

Now, we present how the rate control is used to provide a continuous data flow at the output of the transmission system. The transmission system, as shown in Figure 40, is a particular implementation of the proposed MI-SBTVD system, and has been successfully tested in an FPGA Xilinx Virtex 4 SX35. The simplification has been made in order to present an instructive approach without loss of generality. Besides, this version also does not include RS coding and interleaver and operates under only one transmission mode (64 QAM, guard interval of 1/16, LDPC 3/4, 2048 carriers), providing a throughput of $19.33 \mathrm{Mbps}$.

The basic rate for the entire system is $f_{s}=8.1270$ $\mathrm{MHz}$, which is the sample rate for the baseband modulated signal. In this way, the basic clock has been chosen to be $8.1270 \mathrm{MHz}$ for the overall system. However, some parts of the system also operate with clock 
rates of $4 f_{s}=32.5079 \mathrm{MHz}$ (digital up-converter), $8 f_{s}=$ 65.0159 MHz (some intermediate operations) and $14 f_{s}=$ $113.7778 \mathrm{MHz}$ (LDPC encoder). It is worth showing why the LDPC encoder has been chosen to operate at $14 f_{s}$. Considering only the OFDM Modulation of Figure 40, the throughput is $27.9664 \mathrm{Mbps}$, which is obtained from

$$
\frac{f_{s} \times 6 \times 12248}{2048+128}
$$

in which there are 1248 sub-carriers effectively used for information data, carrying 6 bits each, among a total of 2048 carriers plus 128 "virtual carriers" corresponding to the guard interval of 2048/16 $=128$. The LDPC encoder in its turn can process an average of 1 bit every 5 clock cycles and deliver $4 / 3$ of the input rate, which provides a bit rate of

$$
f_{c l k} \times \frac{4}{3} \times \frac{1}{5} \mathrm{bps},
$$

where $f_{c l k}$ is the operating clock of the LDPC encoder. Following this argument, it can be easily shown that the minimum clock operation for the LDPC encoder is

$$
f_{c l k} \geq 12.9 \times f_{s}
$$

The clock operation for the LDPC encoder was then chosen as $14^{\prime} f_{s}$, including a safety margin to guarantee that the encoder will provide more data than the OFDM Modulation can process, leading to a totally filled Transmission FIFO. In this case, the LDPC input is constantly monitored by the rate control, which provides Null Packets whenever there is no data packet available. When the system detects that the Transmission FIFO is full, then the LDPC encoder is turned off to compensate for its overclocking operation. This process guarantees that data will always be available for the OFDM Modulation. Since the latter operates with a continuous data flow, it cannot stop while there is no data available in the transmission FIFO. This process works well whenever the video data rate is less than or equal to the system throughput, which is $19.33 \mathrm{Mbps}$, ensuring that the input data packet FIFO will never overflow.

Summarizing, the LDPC encoder has been chosen to perform under over-clocking condition, which leads it to be turned off frequently to avoid FIFO overflow. The LDPC input availability in its turn is assured by the rate control scheme, as shown in Figure 39.

\subsection{Channel Estimation}

Coherent modulations like the Alamouti scheme require knowledge of the channel state information (CSI) for correct demodulation of the received signal. In order to obtain an estimate of the CSI, pilot subcarriers are transmitted along with the data, allowing channel estimation at the receiver. In the proposed MI-SBTVD standard,

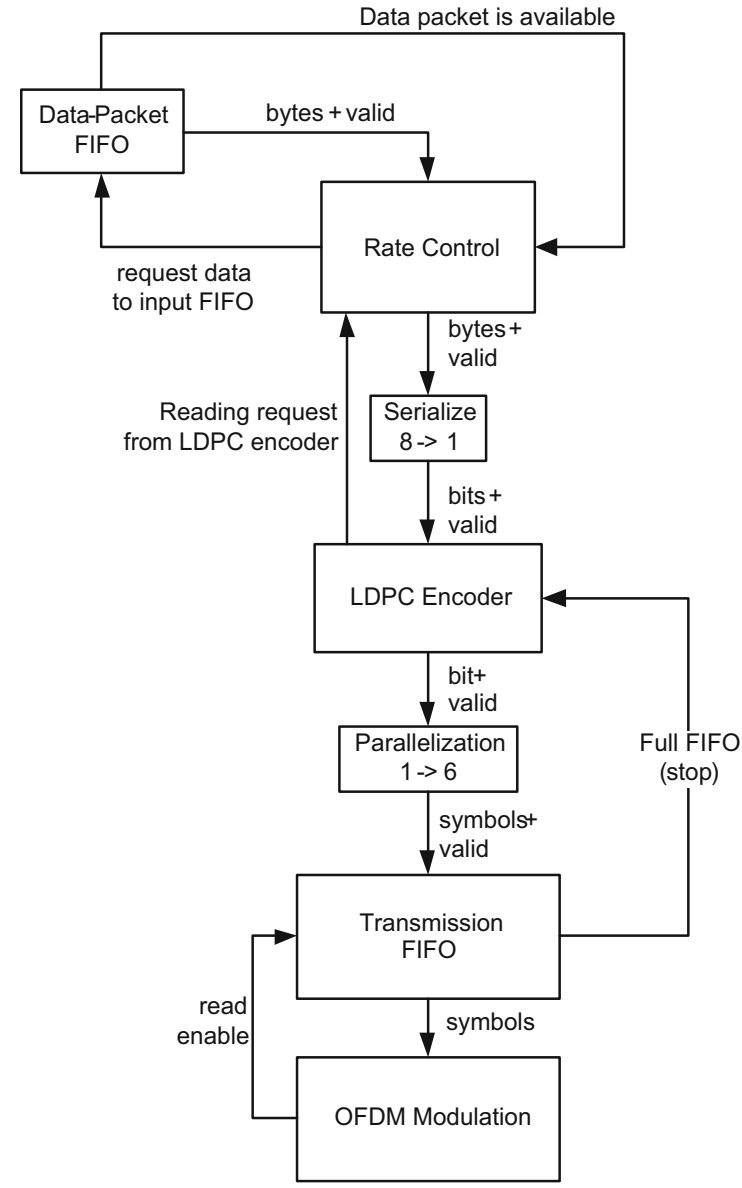

Figure 40. Block diagram showing the data flow for the transmission system.

pilots are positioned 12 subcarriers apart and their positions shifted on 3 subcarriers after each space-time code is transmitted (2 OFDM symbols), as can be seen in Figure 41.

This pilot positioning scheme is very similar to what is done in ISDB-T standard. Such pilot positioning schemes allows bidimensional interpolation to obtain a better resolution of the channel estimates. In the implemented prototype the pilots positioning were static due to its simplicity. The implemented channel estimator is presented in Figure 42 .

The channel estimation task may be split in three minor tasks: the pilot signal generation, the channel estimation at pilot positions and the interpolation. In the prototype, a linear interpolator was chosen due to its simplicity. Each process is briefly explained below, considering the adopted implementation. 


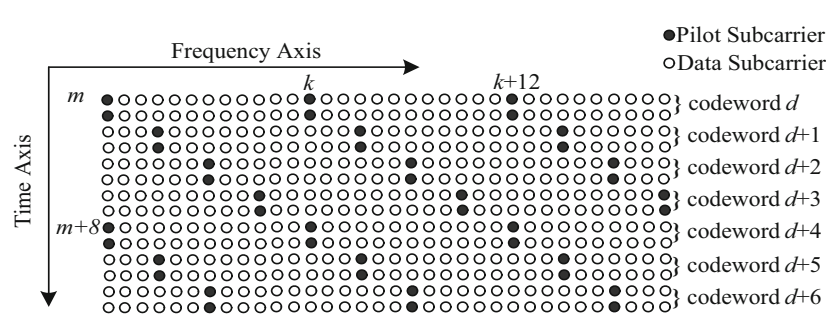

Figure 41. Pilots positioning scheme.

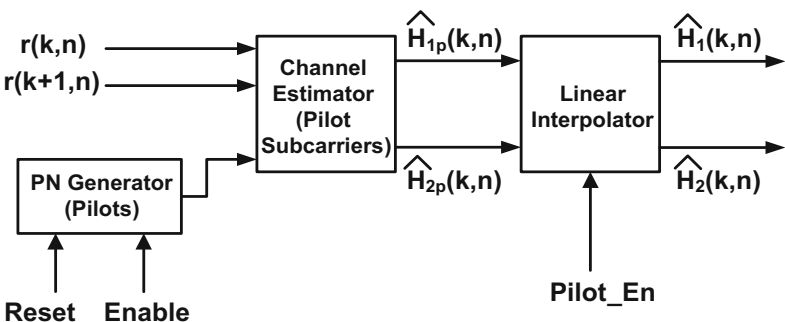

Figure 42. Block diagram of the channel estimation sub-system.

6.3.1. PN Generator: This block is implemented at the transmitter and at the receiver and it generates a pseudo-random sequence that modulates the pilot subcarriers. The PN Generator employed in the MI-SBTVD standard is the same used in ISDB-T and DVB-T, as is the mapping (BPSK). However, in the MI-SBTVD the generated pilots are space-time encoded with the data. This procedure allows the estimation of both channels.

In the implemented receiver prototype, two signals were necessary for correct operation of this block. The enable signal controls the operation of the block, avoiding changes of the PN generator state when invalid data is present at the input. The reset input is responsible to restart the PN generator at the beginning of each OFDM symbol.

6.3.2. Channel Estimator (Pilot Subcarriers): This block performs the calculations described in (10) to obtain the channel state information at the pilot subcarrier positions. As can be seen, the implemented prototype does not use any control signals. It means that a incorrect signal may be present at the output of the channel estimator. However, this problem is negligible because the next block of the prototype only operates with valid information of the channel estimator output. If an incorrect signal is supplied by the channel estimator, the linear interpolator will ignore the signal. Figure 43 presents the block diagram of the channel estimator.
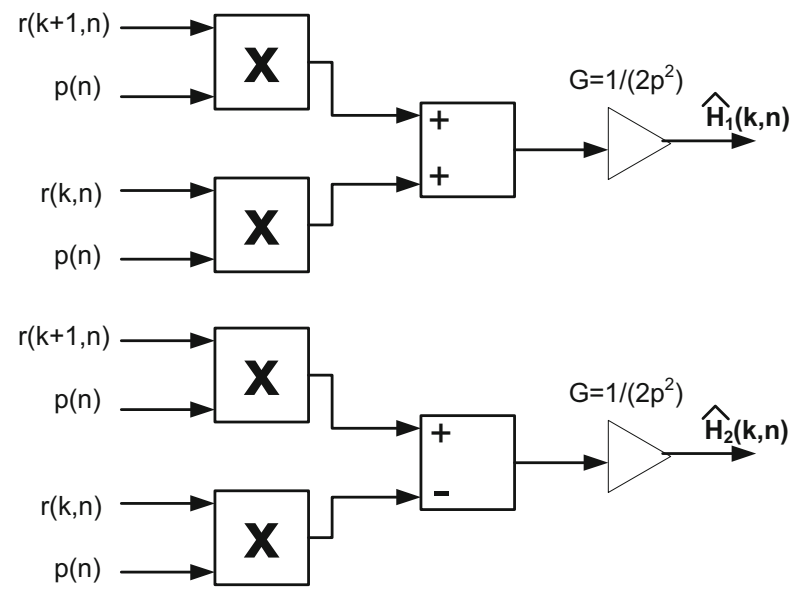

Figure 43. Block diagram of the channel estimator.

6.3.3. Linear Interpolator: The obtained CSI only exists for pilot positions, in which no useful data is transmitted. To obtain an estimation of the CSI in data subcarriers, some kind of interpolator must be employed. Since the adopted pilot positioning for the prototype does not shift in time, there is no reason for adopting a bidimensional interpolation algorithm. Thus, the linear interpolation was chosen due to its simplicity and reasonable performance. The linear interpolator, as its name suggests, calculates the values between two defined points based in a linear function. An example is presented in Figure 44.
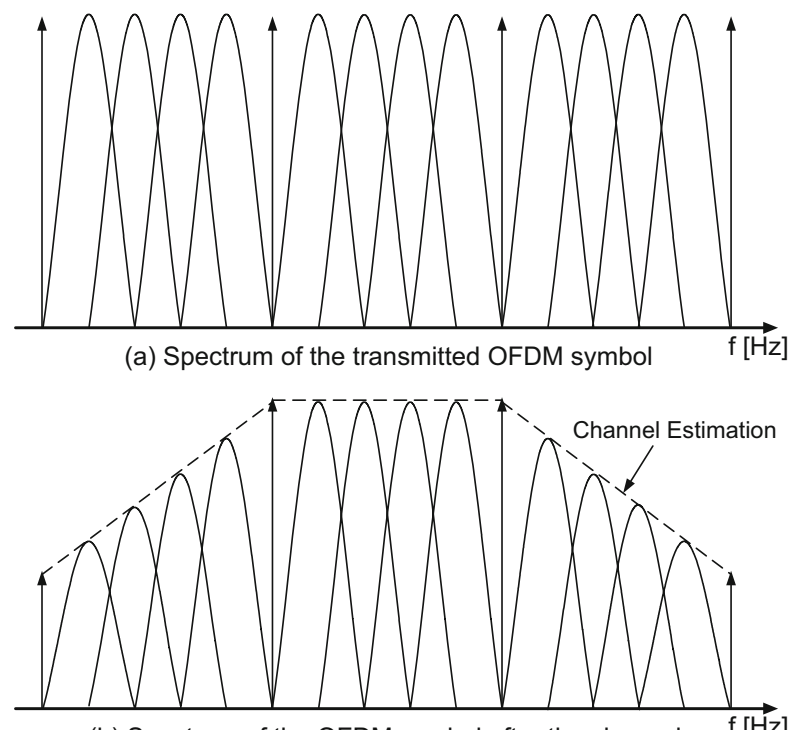

(b) Spectrum of the OFDM symbol after the channel.

Figure 44. Linear interpolation to estimate the channel frequency response.

The estimation of the CSI at a point $p_{1}+\Delta_{n}$ between 
two pilot subcarriers $p_{1}$ and $p_{2}$ is given by:

$$
\hat{H}\left(p_{1}+\Delta_{n}\right)=\frac{\hat{H}\left(p_{2}\right)-\hat{H}\left(p_{1}\right)}{p_{2}-p_{1}} \Delta_{n}+\hat{H}\left(p_{1}\right)
$$

The block diagram of the implemented linear interpolator is presented in Figure 45. The input Pilot_En is only activated when a valid pilot channel estimation is presented in the $H(k, n)$ input. This block has a latency of 12 symbols, since the first valid output is presented only after the next pilot estimate is fed in the input.

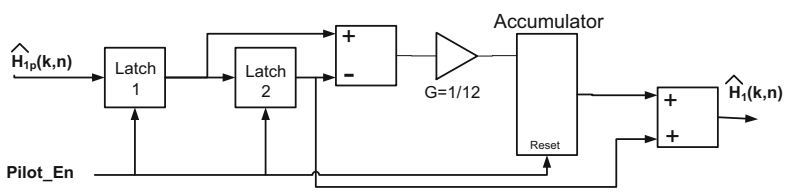

Figure 45. Block diagram of the linear interpolator.

\section{Conclusions}

The system proposed in this paper, as part of the SBTVD Program, was developed in record time thanks to the advanced rapid prototyping tools for DSP algorithms in Field Programmable Gate Arrays (FPGAs). We have shown that the MI-SBTVD presents a significantly improved performance when compared to the current Digital Television Standards. The gains achieved by the MISBTVD vary between $4 \mathrm{~dB}$ and $18 \mathrm{~dB}$, depending on the channel profile, which is a significant achievement. The use of LDPC and STC-OFDM schemes has proved to be an efficient combination of techniques to achieve high throughput at low carrier-to-noise ratio, without a significant increase in receiver complexity.

\section{Acknowledgments}

The authors thank FINEP, INATEL, UNICAMP and UFSC for the financial support.

\section{REFERENCES}

[1] Kenneth P. Davies; HDTV Evolves for the Digital Era; IEEE Communications Magazine, June 1996; pp. 110-112.

[2] "Report 801-4, Part 3"; Reports of the CCIR, 1990, annex to vol. XI, pt. 1, p. 53.

[3] ATSC Document A-54, "Guide to the Use of the ATSC Digital Television Standard", 1995.
[4] European Telecommunication Standard ETS 300 744, "Digital Video Broadcasting (DVB); Framing Structure, Channel Coding and Modulation for Digital Terrestrial Television (DVB-T)", ETSI, 1997.

[5] ARIB, "Terrestrial Integrated Services Digital Broadcasting (ISDB-T). Specification of Channel Coding, Framing Structure and Modulation", September, 1998.

[6] Dayan A. Guimarães and Geraldo G. R. Gomes, "Caracterização do Canal para o MI-SBTVD", Revista Telecomunicações, Dezembro 2006.

[7] ABERT/SET, "Brazilian Tests on Digital Television Systems - Final Report", May, 2000, available in http://www.set.com.br/artigos/testing.pdf.

[8] John Watkinson, MPEG Handbook: MPEG-1, $M P E G-2$ and MPEG-4, 2nd Edition, ButterworthHeinemann, 2004.

[9] Dayan A. Guimarães, Geraldo G. R. Gomes, José G. S. Panaro, Contextualização e delineamento para definição do FEC para o MI-SBTVD. In: SBTVD RFP 2004/18.1.4. Relatório preliminar. Santa Rita do Sapucaí: Instituto Nacional de Telecomunicações (INATEL), 2005. 14 p.

[10] Claude Berrou, A. Glavieux and P. Thitimajshima. Near Shannon limit error-correcting coding and decoding: Turbo-Codes. In: Proceedings of the 1993 Communication Conference, ICC'93, Geneva, Switzerland, pp. 1064-1070, May 1993.

[11] R. Gallager, Low-Density Parity-Check Codes. PhD Thesis, Cambridge, MIT Press, 1963.

[12] Geraldo G. R. Gomes; Dayan A. Guimarães, Tarciano. F. Pegoraro, Definição de códigos, entrelaçamento e modulação. In: SBTVD RFP 2004/18.1.4. Santa Rita do Sapucaí: Instituto Nacional de Telecomunicações (INATEL), 2005. 14 p.

[13] S. Benedetto and E. Biglieri, Principles of Digital Transmission: With Wireless Applications, Plenum Pub Corp, pag. 232, 1999.

[14] S. Alamouti, "A simple transmit diversity technique for wireless communications", IEEE J. Select. Areas Commun., vol. 16, no. 8, pp. 1451-1458, Oct. 1998.

[15] M. Yacoub, Foundations of Mobile Radio Engineering, CRC Press, 1993.

[16] Luciano L. Mendes, Análise de Desempenho de Sistemas Multiportadora em Canais com Ceifamento de Pico, Master Thesis, INATEL, 2003. 
[17] Gustavo C. Lima and Luciano L. Mendes, "Performance of STC-OFDM and SFC-OFDM for Digital Television Broadcasting", GSPx'06, Amsterdam, 2006.

[18] Ye Li, "Pilot-symbol-Aided Channel Estimation for OFDM in Wireless Systems," IEEE Trans. Vehic. Tech, Vol. 49, No.4, pp. 1207-1215, 2000.

[19] A. R. Bahai and B. R. Saltzberg, Multi-Carrier Digital Communications - Theory and Applications of OFDM, Kluwer Academic, 1999.

[20] K. F. Lee and D. B. Willians, "A Space-Time Coded Transmit Diversity Technique for Frequency Selective Fading Channels", IEEE Sensor Array and Multichannel Signal Processing Workshop, pp. 149-152 Cambridge, MA, March 2000.

[21] K. F. Lee and D. B. Willians, "A Space-Frequency Transmit Diversity Technique for OFDM Systems", IEEE Globecom 2000, Vol. III, pp. 1473-1477, San Francisco, USA, November 2000.
[22] José M. C. Brito, José S. G. Panaro, Luciano L. Mendes, Dayan A. Guimarães, Geraldo G. R. Gomes e Fabbryccio A. C. M. Cardoso, Relatório de Prova de Conceito, Relatório Técnico, Convênio 0026/05, MI-SBTVD, October 2005.

[23] Mackenzie/Abert/Set, Relatório Final, Testes em Sistemas de TV Digital, Descrição Geral dos Testes de Laboratório (in Portuguese).

[24] W. Tuttlebee, Software Defined Radio: Enabling Technologies, John Wiley \& Sons, NY, USA, 2002.

[25] R. E. Crochiere and L. R. Rabiner, Multirate Digital Signal Processing, Prentice Hall Signal Processing Series, Alan V. Oppenheim Editor, New Jersey, USA, 1983.

[26] Tarciano Pegoraro et al, Design, Simulation and Hardware Implementation of a Digital Television System: LDPC channel coding, IEEE International Symposium on Spread Spectrum Techniques and Applications - ISSSTA-2006, Manaus, Brazil, August, 2006. 